\title{
Mosaic and non-mosaic pedh19 mutation leads to neuronal hyperexcitability in zebrafish
}

\author{
Barbara K. Robens',2, Xinzhu Yang,, Christopher M. McGraw ${ }^{1,2,3,4}$, Laura H. \\ Turner $^{1,2}$, Carsten Robens5, Summer Thyme $^{6}$, Alexander Rotenberg,3,7, Annapurna \\ Poduri', ${ }^{1,2,3,7}$
}

${ }^{1}$ Department of Neurology, F.M. Kirby Neurobiology Center, Boston, MA, USA

Epilepsy Genetics Program, Department of Neurology, Boston Children's Hospital, Boston, MA, USA.

3Department of Neurology, Harvard Medical School, Boston, MA, USA

4Division of Epilepsy, Department of Neurology, Massachusetts General Hospital, Boston, MA, USA

5MIT-Harvard Center for Ultracold Atoms, Research Laboratory of Electronics, and Department of Physics, Massachusetts Institute of Technology, Cambridge, Massachusetts 02139, USA

${ }^{6}$ Department of Neurobiology, University of Alabama at Birmingham, Birmingham, AL, USA

7Division of Epilepsy and Clinical Neurophysiology, Department of Neurology, Boston Children's Hospital, Boston, MA, USA

Correspondence should be addressed to Annapurna.Poduri@childrens.harvard.edu

\begin{abstract}
Epilepsy is one of the most common neurological disorders. The X-linked gene PCDH19 is associated with sporadic and familial epilepsy in humans, typically with early-onset clustering seizures and intellectual disability in females but not in so-called 'carrier' males, suggesting that mosaic PCDH19 expression is required to produce epilepsy. To characterize the role of loss of PCDH19 function in epilepsy, we generated zebrafish with truncating pcdh19 variants. We observed hyperexcitability phenotypes in both mosaic and non-mosaic $p c d h 19^{+/-}$and ${ }^{-/}$mutant larvae, indicating that Pcdh19 cellular mosaicism is not required for network hyperexcitability in zebrafish. Further, zebrafish with non-mosaic pcdh19 mutation display reduced numbers of inhibitory interneurons and transcriptional down-regulation of key inhibitory synapse components, suggesting a potential cellular basis for the observed hyperexcitability. Our findings in both mosaic and non-mosaic pcdh19 mutant zebrafish challenge the prevailing theory that mosaicism governs all PCDH19-related phenotypes and point to interneuron-mediated mechanisms underlying these phenotypes.
\end{abstract}




\section{Introduction}

Epilepsy is a common chronic disorder of the brain characterized by recurrent, unprovoked seizures affecting all age groups, with a substantial contribution from genetic causes. The X-linked gene PCDH19, encoding the cell-cell adhesion molecule protocadherin 19, has emerged as one of the most prominent single genes associated with epilepsy (Dibbens et al., 2008; Duszyc et al., 2015; Epi4K Consortium and Epilepsy Phenome/Genome Project, 2017). Pathogenic variants in PCDH19 were shown to cause PCDH19-clustering epilepsy (CE), previously referred to as Epilepsy and Mental Retardation Limited to Females (EFMR), OMIM \#300088). Disease manifestations vary in severity, even among individuals with the same variant. They include earlyonset seizures that are often fever-related and cluster with multiple seizures occurring one after another, intellectual disability, and autism (Dibbens et al., 2008; Dimova et al., 2012; Smith et al., 2018). The disorder follows a unique inheritance pattern, affecting primarily heterozygous females and sparing hemizygous males, including fathers of affected girls who carry the same pathogenic variant, at least from overt epilepsy and severe symptomatology; rarely observed males who are mosaic for hemizygous variants in this gene also display these major symptoms (Kolc et al., 2018). It has been proposed that the tissue mosaicism caused by random Xinactivation in all female cells leads to an abnormal cellular pattern during development, and that mosaic males follow a similar pattern. The so-called 'cellular interference' hypothesis posits that the presence of a mixture of cells in the brain, expressing either wild-type or mutant PCDH19 protein, leads to abnormal brain development and is consequently the underlying cause of the clinical manifestations (Depienne et al., 2009). However, it is still unclear how mosaic expression of PCDH19 in the brain leads to epileptogenesis and whether mosaic expression of wild-type (WT) and mutant protein in neurons is the sole cause for the clinical manifestations of PCDH19-CE.

The PCDH19 gene consists of 6 exons, and most of the over 100 identified patient variants occur in exon 1, the largest exon, which encodes the 6 extracellular cadherin domains (Figure 1) (Kolc et al., 2018). PCDH19 is highly expressed in the brain and belongs to the largest subgroup of cadherins that are involved in calcium-dependent cell-cell adhesion (Depienne and LeGuern, 2012). However, the exact function of PCDH19 is still largely unknown. Recent studies in mice suggest that PCDH19 determines cell adhesion affinities and is involved in cell sorting during cortical development and mossy fiber synapse development (Hoshina et al., 2021; Pederick et al., 2018). Even though Pcdh19 heterozygous mice do not show gross behavioral abnormalities or spontaneous seizures, treatment with the $\gamma$-aminobutyric acid type A (GABA-A) receptor inhibitor flurothyl revealed increased seizure susceptibility; interestingly, this finding does not require tissue mosaicism as it is present in both female heterozygous and homozygous mice (Hayashi et al., 2017; Pederick et al., 2016; Pederick et al., 2018; Rakotomamonjy et al., 2020). Other in vitro studies suggest a role for Pcdh19 in establishing proper brain architecture and neuronal connections (Mincheva-Tasheva et al., 2021; Pederick et al., 2016). Similar roles have been proposed for the highly conserved zebrafish (Danio rerio) Pcdh19 protein which was shown to be involved in cell proliferation and neuronal organization of the optic tectum, the largest region of the zebrafish brain, important for visual processing (Cooper et al., 2015).

To better understand the role of PCDH19 in the developing brain and the impact cellular Pcdh19 mosaicism has in neurodevelopmental epilepsy, we developed mosaic and non-mosaic models of PCDH19 disorder in zebrafish by CRISPR/Cas9-mediated gene editing. Importantly, the mosaic 
mutants are comprised of some cells expressing mutant pcdh19 and some wildtype, most closely approximating the mosaic expression that occurs in heterozygous female patients with PCDH19CE. Because pcdh19 is not present on a sex chromosome in zebrafish, we had the opportunity to also create mutants with germline heterozygous and homozygous frameshift mutations, predicted to lead to premature truncation and consequent loss of one or both copies of $p c d h 19$. This provides the unique opportunity to evaluate the effects of mosaic and non-mosaic pcdh19 variants in the zebrafish system, which is well-suited to evaluate features of epilepsy and neurodevelopmental dysfunction (Griffin et al., 2017). Given the human disease presentation of PCDH19-CE with seizures (spontaneous and fever-induced), learning defects, and other neuropsychiatric features, we assayed our models for zebrafish correlates of these features. We also performed neuroanatomic and transcriptomic analyses to understand the mechanisms by which both mosaic and non-mosaic Pcdh19 loss-of-function (LOF) may lead to phenotypes in larval zebrafish.

\section{Results}

\section{Generation of pcdh19 zebrafish mutants.}

We successfully generated zebrafish mutant lines with frameshift mutations that result in premature stop codons in exon 1 of the pcdh19 gene (Figure 1A). We studied stable heterozygous ( $p c d h 19^{+/-)}$and homozygous ( $p c d h 19^{-/-}$) knockout (KO) zebrafish lines as well as mosaic $\mathrm{F}_{\mathrm{o}}$ larvae that were acutely injected with the same guide RNA targeting the first exon of pcdh19, which results in a mosaic expression pattern of Pcdh19 across all Pcdh19-expressing cells. Presence of the mosaic mutation was verified by Sanger sequencing of DNA from pcdh19 sgRNA-injected $F_{0}$ larvae (after the experiments described below), and a 'knockout score' (reflecting the percentage of cells bearing the variant) was quantified by Interference of CRISPR Edits (ICE) analysis (Figure 1B). We observed highly variable numbers of indels generated by the CRISPR/Cas9 approach, which we leveraged in this study to mimic the tissue mosaicism present in females with PCDH19 variants caused by random X-inactivation events during fetal development. Mosaic mutants with KO scores of $>40$ were used for subsequent experiments. Successful loss of full-length Pcdh19 protein in different knockout mutants was verified by Western blot (Figure 1C). These different Pcdh19 LOF models reflect models of the human condition and give us the unique opportunity to study the effects of mosaic and non-mosaic Pcdh19 disruption (Figure 1D). None of our generated pcdh19 mutant fish lines showed gross morphological abnormalities. Head and body size and shape were normal, general mobility, fertility, and survival (to at least 2 years) were intact. 

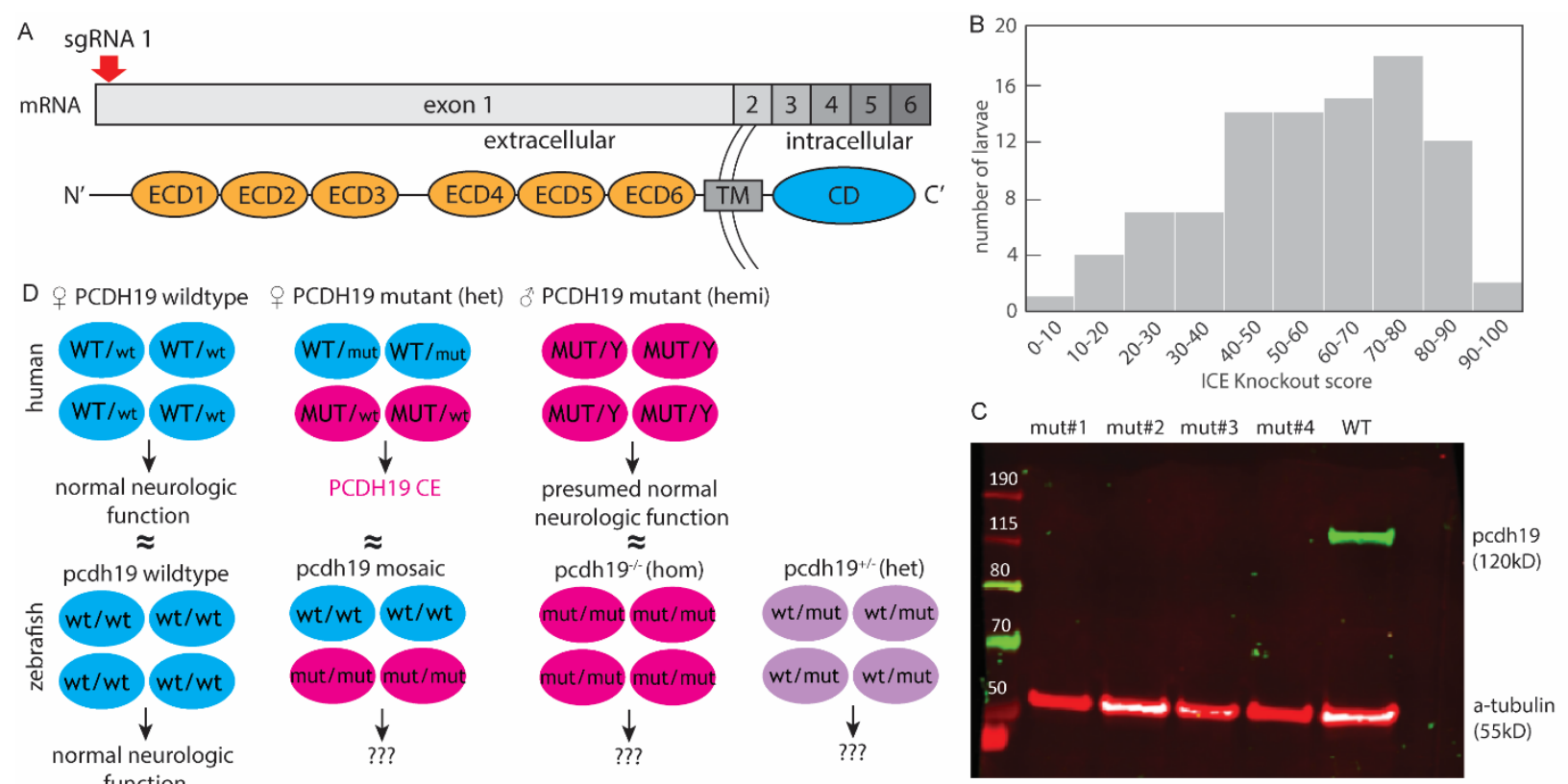

Figure 1: Mutant pcdh19 loss-of-function zebrafish model. (A) Mutations introducing a 44-nucleotide insertion and 1nucleotide deletion were introduced in the pcdh19 gene at exon 1 (coding for the extracellular cadherin domains, ECD) (TM, transmembrane domain; CD, C-terminal domain), resulting in a frameshift. (B) Histogram of 192 Fo larvae injected with pcdh19 sgRNA illustrating the distribution of the KO score, representing the proportion of cells predicted to result in the knockout due to the presence of a frameshift. (C) Western blot showing loss of full-length Pcdh19 protein in four different generated $p c d h 19^{-/-}$mutant lines while WT larvae have intact Pcdh19 protein expression. (D) Schematic to illustrate the proposed disease mechanism in male and female humans and how we approached modeling them in zebrafish (wt, wildtype; mut, mutant).

\section{Increased spontaneous firing activity in zebrafish larvae with pcdh19 mutations.}

To determine whether mosaic or non-mosaic pcdh19 mutations result in abnormal neuronal network activity, we measured local field potentials (LFPs) in the optic tectum of 6-7dpf zebrafish larvae (Figure 2), a brain region known to have abnormal neuronal organization in the setting of Pcdh19 dysfunction (Cooper et al., 2015). In general, we distinguished between two types of spiking events that occurred in all our recordings at varying frequencies: events with a narrow peak and small amplitude (alpha events) and events with a broad peak and large amplitude (beta events) (Figure 2A). LFP traces of wildtype larvae only occasionally captured the high-voltage beta events (approx. 0.2-0.4mV), while KO mutants displayed a range of epileptiform abnormalities. We often observed medium- to high- voltage deflections clustered in bursts and sometimes also in isolation. Discrete epileptiform discharges were 150-500ms long and resulted in 0.2-0.3mV sharp or blunt deflections. Quantification revealed a significantly increased beta spike rate in mosaic and non-mosaic mutant pcdh19 lines compared to their respective controls, while the three different control groups showed a uniformly low beta spike rate (Figure 2B). Beta event amplitude was significantly increased only in non-mosaic $p c d h 19^{+/-} \mathrm{KO}$ larvae (Figure 2C). Quantification of burst frequency revealed abnormal clustering of large spiking events in the mosaic and non-mosaic $p c d h 19^{+/-}$mutants compared to control larvae (Figure 2D). Similar but less frequent epileptiform abnormalities were observed in homozygous $p c d h 19^{-/-}$zebrafish larvae. We confirmed these abnormal electrographic patterns in the form of increased and clustered bursts of abnormal neuronal firing in heterozygous and homozygous larvae in an independent 
pcdh19 KO line with a frameshift mutation introduced at exon 2 (Suppl.Fig1A-D). These results indicate that a disruption of Pcdh19 function results in increased neuronal activity in pcdh19 mutant fish, independent of mosaicism.

A

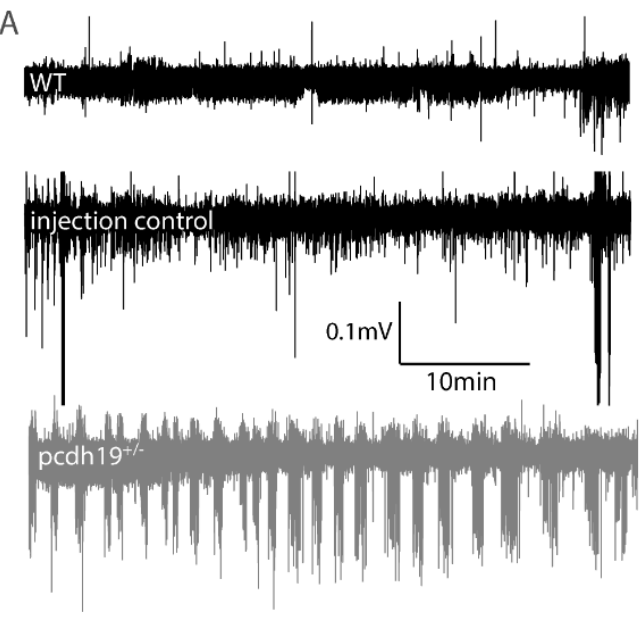

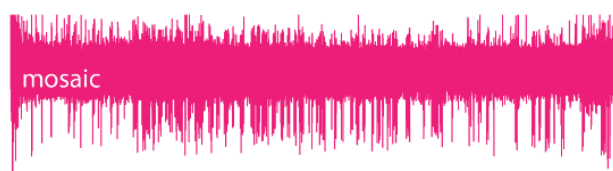
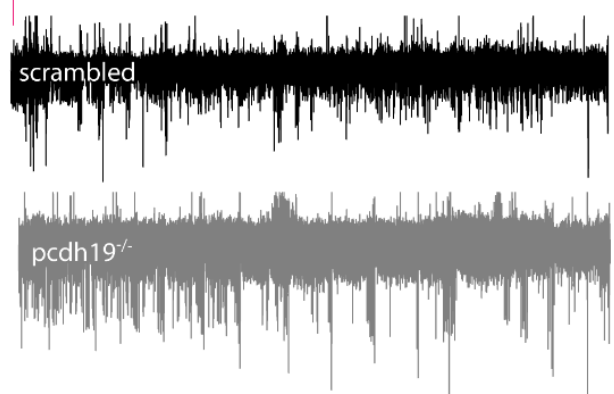
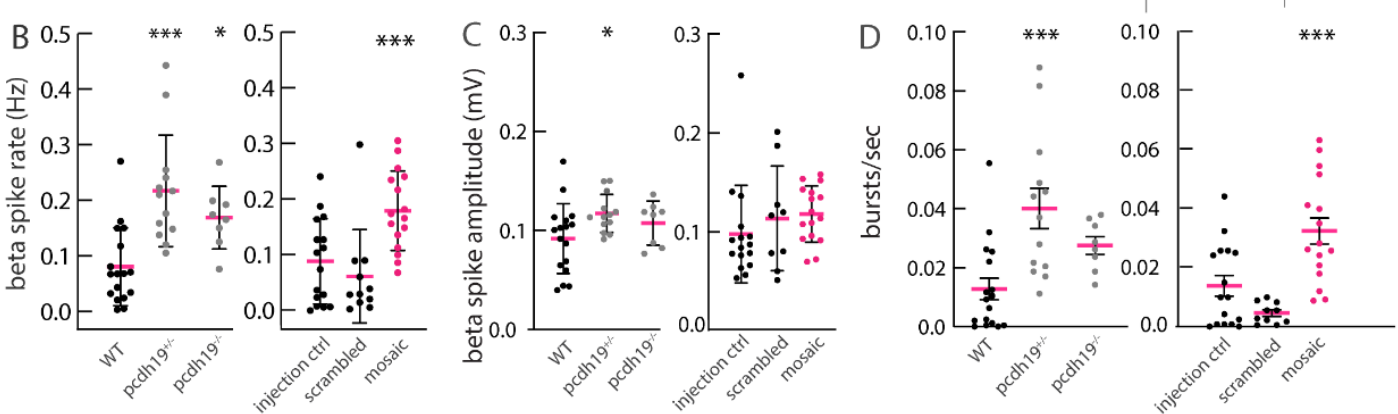

Figure 2: Hyperexcitability in LFP recordings of pcdh19 mutant larval tectum. (A) Exemplary traces of LFP recordings for control and pcdh19 mutants showing beta events consisting of bursts of spikes present in pcdh19 mutants, which are rare in controls. (B) Quantification shows an increase in beta event rate in all pcdh19 mutant lines, compared to their respective controls; mosaics were compared to scrambled injected and a 'control injected' group representing larvae that were injected with pcdh19 sgRNA but had $<10 \%$ indel frequencies. (C) The average beta spike amplitude is increased in pcdh19+/- KO mutant lines compared to WT controls. (D) The average beta burst frequency is increased in pcdh19+/- KO and mosaic mutants compared to their respective controls. $\mathrm{n}=16 \mathrm{WT}, \mathrm{n}=13 \mathrm{pcdh} 19^{+/-}, \mathrm{n}=8 \mathrm{pcdh} 19^{-/-}, \mathrm{n}=16$ mosaic, $n=15$ injection control, $n=11$ scrambled injected. One-way ANOVA with Dunnett's multiple comparisons test, ${ }^{*} \mathrm{p}<0.05,{ }^{* *} \mathrm{p}<0.01,{ }^{* * *} \mathrm{p}<0.001$.

In complementary experiments, we further tested for hyperexcitability as a result of pcdh19 dysfunction. First, we used the genetically encoded calcium sensor GCaMP6s to assess whole brain activity using lightsheet microscopy (Turrini et al., 2017). Cytosolic GCaMP6s is expressed pan-neuronally (elav3 promoter) labelling cell bodies and neuropil in the central nervous system (CNS) of larval zebrafish and fluorescence increases in response to calcium influx during neuronal activity (Figure 3A). We observed abnormal network activity in the tectum of a Pcdh19 ${ }^{+/-} \mathrm{KO}$ mutant via calcium imaging, showing distinct clusters of neurons in the tectum with short bursts of increased activity (Figure 3B). We also observed longer-duration periods of dramatically increased whole brain activity (Figure 3C,D) lasting 1 to 2 min (Suppl. Video 1). Second, we used the neuronal activity marker phospho-ERK (pERK) to visualize and identify brain areas with 
increased neuronal baseline activity 10 min prior to death (Thyme et al., 2019). Whole brain antibody staining against pERK relative to total ERK (tERK) revealed increased pERK fluorescence signals within different parts of the tectum mainly in mosaic mutants and to a lesser extend in $p c d h 19^{-/-}$mutants relative to control animals. While mosaic mutants primarily showed an increase in pERK signal in the neuropil, where neuronal processes converge, homozygous mutants had a decrease in parts of the medial tectum (Figure $3 \mathrm{E}$ ); and heterozygous mutants showed no difference vs. controls. To corroborate that the increased brain activity in mosaic fish can be attributed to Pcdh19 dysfunction, we generated mosaic pcdh19 mutants using a different sgRNA targeting pcdh19 at exon 2. We observed a similar pattern of increased pERK signal in the tecum of these larvae (Suppl.Fig1E). These experiments indicate that abnormal brain activity might be limited to the optic tectum in larval zebrafish. Together, these results provide further evidence that Pcdh19 dysfunction results in altered brain activity.
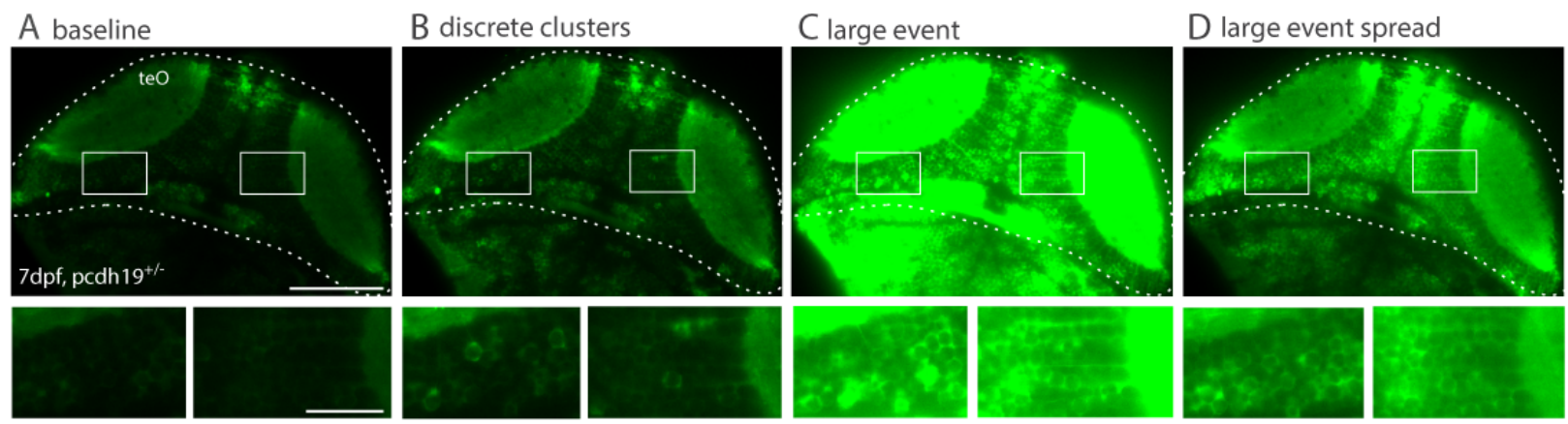

E increased signal (rel. to control)

decreased signal (rel. to control)
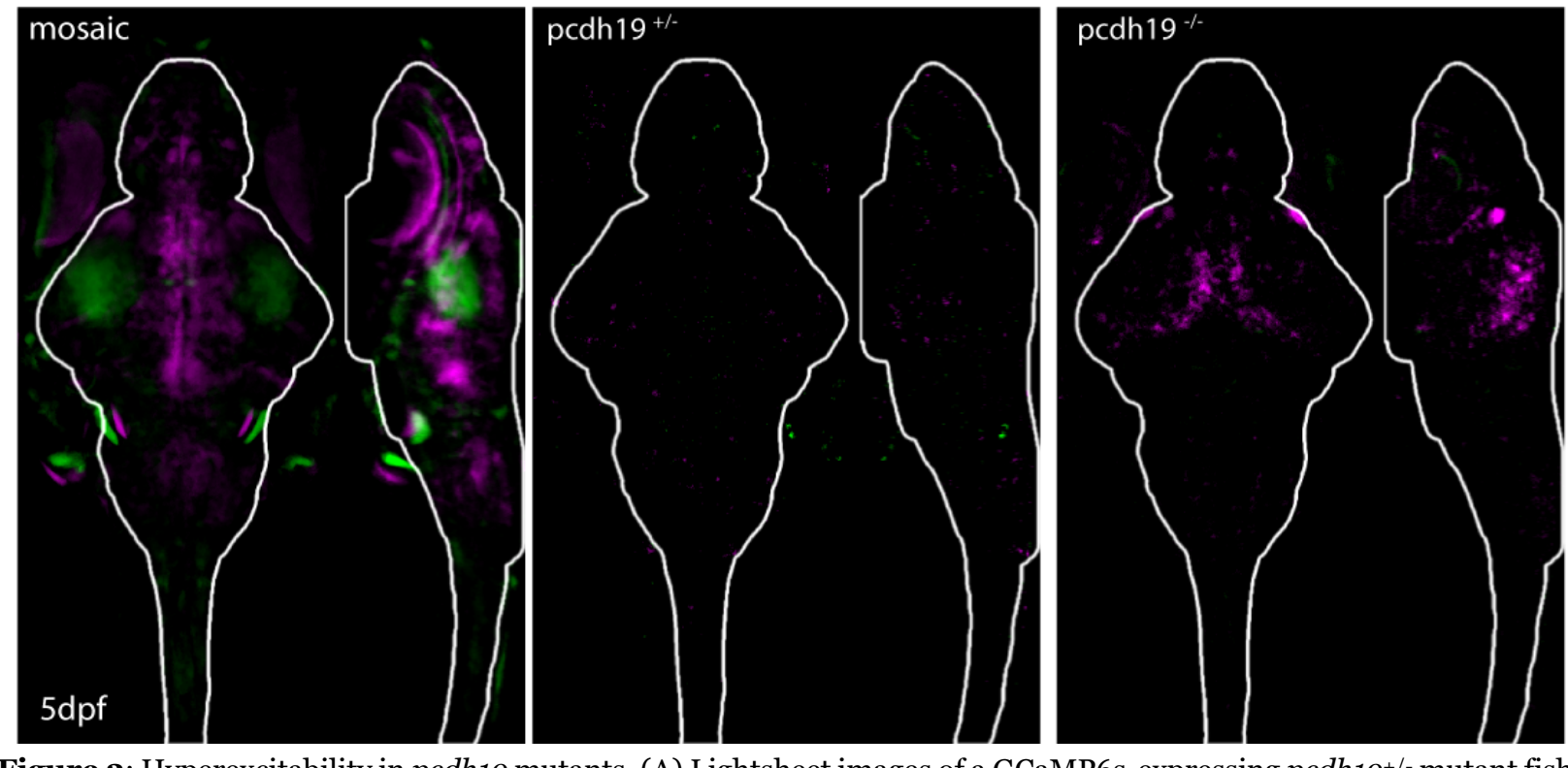

Figure 3: Hyperexcitability in pcdh19 mutants. (A) Lightsheet images of a GCaMP6s-expressing $p c d h 19^{+/-}$mutant fish at baseline. Scale bar 10oum and 20um in higher magnification images below. teO; tectum opticum. (B) Increased GCaMP fluorescence in two different neuronal clusters of the tectum. (C) Dramatic increase in GCaMP fluorescence across the entire brain of a $p c d h 19^{+/-}$mutant fish during a large discharge event. (D) Slowly attenuating GCaMP intensity after the large event. (E) Sum of slices projection of confocal images show pERK signal intensity is increased in retinotectal processes in mosaic pcdh19 mutant larvae, no change in pcdh19//- mutants, and decreased signal in pcdh19-/- mutants, $\mathrm{n}=18$ mosaic, $\mathrm{n}=28$ scrambled injected, $\mathrm{n}=82 \mathrm{pcdh} 1 \mathrm{9}^{+/-}$and $\mathrm{n}=28 \mathrm{pcdh} 19^{-/-}$larvae. 


\section{Mosaic and non-mosaic mutations in pcdh19 do not lead to a consistent seizure phenotype.}

To assess whether the abnormal patterns of electrical activity observed in the LFP recordings and in calcium imaging correlate with a tendency toward seizure-like activity, we subjected mutant and control larvae to high-throughput assays to further characterize the range of abnormalities resulting from Pcdh19 dysfunction. We evaluated for behavioral seizure-like events in zebrafish larvae, defined as short episodes of hyperlocomotion that result in rapid swimming (Baraban et al., 2013) that can either occur spontaneously or can be evoked by proconvulsant compounds or temperature elevation (hereafter referred to as seizures). At baseline, we observed only occasional seizures in WTs (2.5\%), which was comparable to the $p c d h 19^{+/-}(2 \%), p c d h 19^{-/-}$(0.7\%), and mosaic (0.3\%) groups (Figure 4A). When challenged with a concentration of PTZ (0.1 mM) that is not sufficient to generate seizures in WT larvae (a 'subthreshold' concentration), a significantly higher proportion of $p c d h 19^{+/-}$mutant larvae exhibited seizures than WT larvae (4.2\% vs 0.9\% WT) (Figure 4A). Seizing pcdh19//- mutants also had more seizures over the duration of the trial than the WT larvae (Figure 4A). Increasing the bath temperature, and consequently larval body temperature, from $22^{\circ} \mathrm{C}$ to $37^{\circ} \mathrm{C}$ over the course of 20 min resulted in a few seizures in WT larvae $(<2 \%)$ and no significant changes from the baseline seizure frequency in the pcdh19 mutant groups: $p c d h 19^{+/-}$(3.1\%), pcdh19-/- (1.2\%), mosaic pcdh19 mutants (4.6\%) (Figure 4A). Increasing the PTZ concentration to $2.5 \mathrm{mM}$, a concentration at which most animals are expected to develop seizures, significantly more $p c d h 19^{+/-}$larvae (99.5\%) had at least one seizure compared to WT larvae (90.8\%) (Figure 4B). The average number of seizures in each group was similar $(13.1 \%$ seizures/WT vs $13.7 \%$ seizures/pcdh19+/- larvae) (Figure 4B). In some forms of epilepsy, seizures can be triggered by intermittent photic stimulation; we therefore challenged mutant larvae with

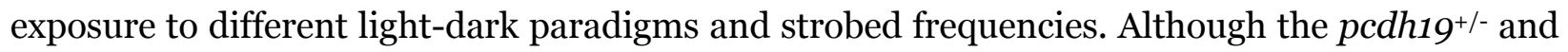
pcdh1 $9^{-/-}$KO mutants moved on average more and with higher maximum velocities during dark periods, we did not detect striking differences or seizures in response to different strobe light frequencies between groups (Figure 4D). Thus, we conclude that only the non-mosaic heterozygous pcdh19 mutant larvae showed a slightly increased susceptibility to provoked seizures unlike the mosaic and non-mosaic $p c d h 19^{-/-}$mutant larvae and that the electrographic abnormalities observed during LFP recordings in all of the lines do not correlate with a gross behavioral seizure phenotype.

Since our behavioral seizure detection and analysis might not be sensitive enough as a readout to capture the hyperexcitability phenotype of pcdh19 mutants, we performed low-resolution, highthroughput calcium imaging to assess for and characterize seizure-like activity. We observed pcdh1 $9^{+/-}$and pcdh19 ${ }^{-/-}$larvae with stereotypical rapid, swirling swim behaviors and a simultaneous increase in GFP fluorescence, likely representing seizures with increased brain activity that subsided after the events (Suppl. Video 2, 3). However, quantification with our event detection algorithm did not capture a difference in the overall rate of high calcium events between untreated pcdh19 KO mutants and WT or scrambled injected vs mosaic animals (Figure 4C). Treatment with 10mM PTZ lead to a significant increase in calcium events compared to the untreated larvae, however this increase was similar in pcdh19 KO mutants and controls. Interestingly, PTZ treatment provoked on average slightly more calcium events in mosaic pcdh19 
mutants compared to scrambled injected controls (Figure 4C). These results suggest that lowresolution microscopy may not be sensitive enough to visualize changes in neuronal firing patterns that occur in all $p c d h 19^{+/-}$mutant lines, given the low number of synchronously firing neurons within a small brain area, as suggested in Figure $3 \mathrm{~B}$.
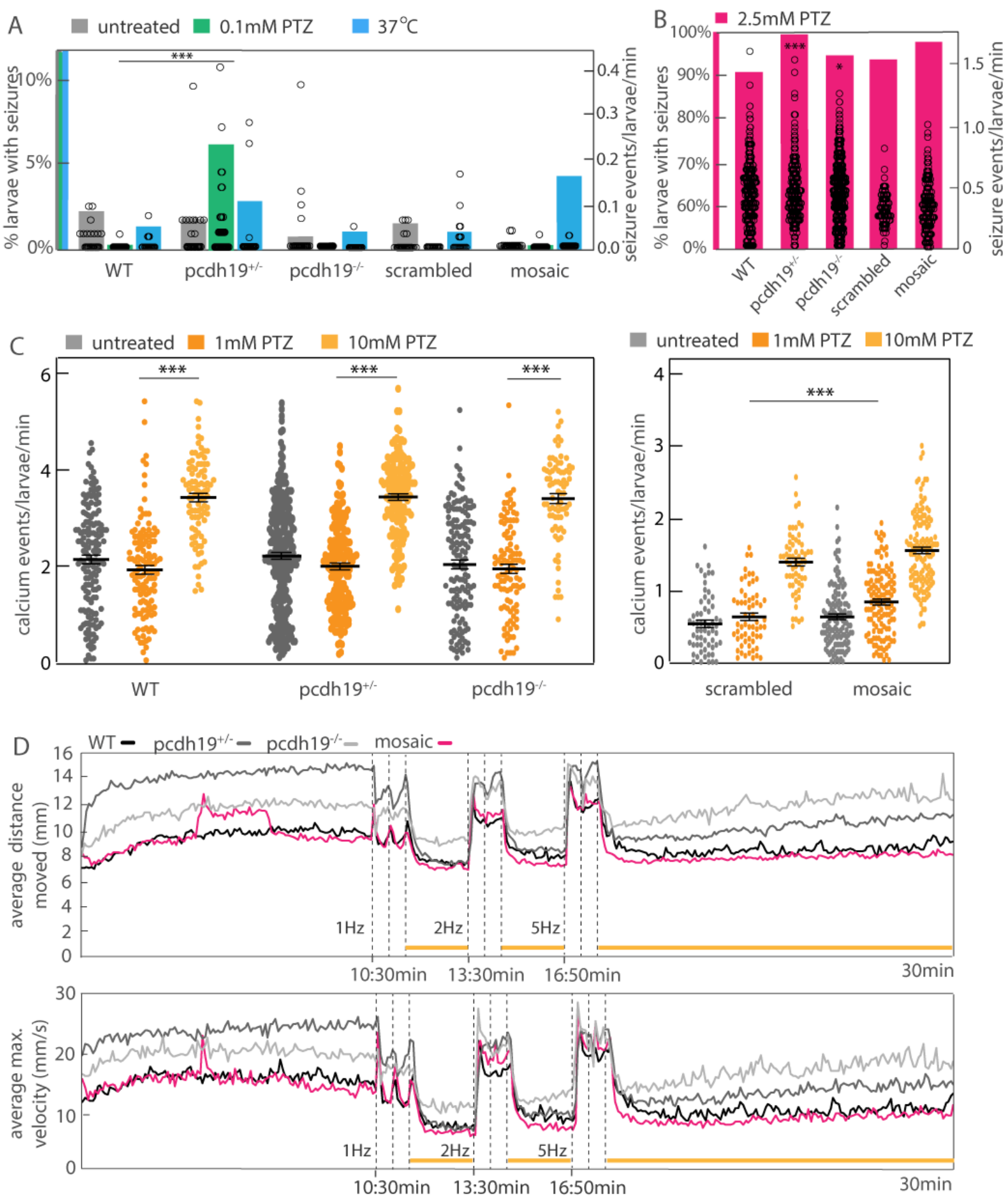
Figure 4: High-throughput detection of spontaneous and provoked seizure activity by behavioral and calcium fluorescence-based assays. (A) Quantification of the percentage of larvae with seizure events (left axis, green bar $=0.1 \mathrm{mM}$ PTZ exposure blue bar $=$ exposure to $37^{\circ} \mathrm{C}$ warm water) and the number of seizures per larvae per minute (right axis, open circles) untreated (grey bars) or after exposure to o.1mM PTZ (green bars) or temperature increase up to $37^{\circ} \mathrm{C}$ (blue bars). pcdh19+/- larvae treated with o.1mM PTZ showed an increased number of seizures compared WT.

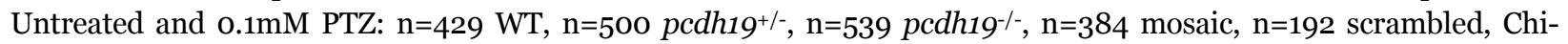
squared test, ${ }^{* * *} \mathrm{p}<0.001$. Untreated and temperature elevation: $\mathrm{n}=258 \mathrm{WT}, \mathrm{n}=96 \mathrm{pcdh} 19^{+/-}, \mathrm{n}=163 \mathrm{pcdh} 19^{-/-}, \mathrm{n}=150$ mosaic, $n=192$ scrambled, Chi- squared test, ${ }^{*} \mathrm{p}<0.05,{ }^{* * *} \mathrm{p}<0.001$. (B) Quantification of seizure events in larvae treated with 2.5mM PTZ (left axis, pink bars=2.5mM PTZ) induces seizures in almost all $p c d h 19^{+/-}$and pcdh19/- mutant larvae, significantly more than in WT larvae. $\mathrm{n}=447 \mathrm{WT}, \mathrm{n}=225 \mathrm{pcdh}^{\mathrm{s}} \mathrm{9}^{+/-}, \mathrm{n}=620 \mathrm{pcdh} 1 \mathrm{9}^{-/-}, \mathrm{n}=192 \mathrm{mosaic}, \mathrm{n}=192 \mathrm{scrambled}$, Chi- squared test, ${ }^{*} \mathrm{p}<0.05,{ }^{* * *} \mathrm{p}<0.001$. The frequency of seizures in each animal is similar across genotypes (right axis, open circles) (C) Quantification of the average event rate of calcium transients in GCaMP6s larvae shows no difference between WT and mutants in the untreated condition; there is a significant increase in events in all groups after exposure to 10mM PTZ, similar to WT. Comparing mosaic and scrambled injected larvae shows a significant increase in calcium events when treated with $1 \mathrm{mM}$ PTZ. $\mathrm{n}=155 \mathrm{WT}, \mathrm{n}=264 \mathrm{pcdh} 1 \mathrm{9}^{+/-}, \mathrm{n}=145 \mathrm{pcdh}$ 19/-, $\mathrm{n}=128 \mathrm{mosaic}$, $\mathrm{n}=61$ scrambled, one-way ANOVA with Tukey's multiple comparisons test for Pcdh19 KO mutants and two-way ANOVA with Sidak's multiple comparisons test mosaic vs scrambled and PTZ vs no treatment, ${ }^{*} \mathrm{p}<0.05,{ }^{* * *} \mathrm{p}<0.001$. (D) Average movement (distance moved in top panel and maximum velocity in lower panel) of larvae exposed to dark, $100 \%$ light (indicated by horizontal yellow lines), or 3 pulses, $3 \mathrm{sec}$ each, of strobe light $(1 \mathrm{~Hz}, 2 \mathrm{~Hz}$, and $5 \mathrm{~Hz}$, indicated by vertical dashed lines) over 30 min did not change in response to the different light paradigms in pcdh19 mutants compared to WT. $\mathrm{n}=223 \mathrm{WT}, \mathrm{n}=\mathbf{2 2 7} \mathrm{pcdh} 19^{+/-}, \mathrm{n}=134 \mathrm{pcdh} 19^{-/-}, \mathrm{n}=261$ mosaic.

\section{Fewer inhibitory neurons in the tectum of pcdh19 $^{+/-}$mutant larvae.}

To probe for a structural defect that might explain the abnormal neuronal excitability in pcdh19 mutant larvae in the transgenic background $\mathrm{Tg}(\mathrm{dl}$ x6a-1.4kbdlx5a/dlx6a:GFP::vGlut:DsRed), we aimed to determine the number of inhibitory and excitatory neurons present in mutants vs. WT conditions. At 5dpf, WT transgenic larvae express GFP in distinct neuronal populations clustering in the tectum, forebrain, and midbrain, while dsRed expression is found in all brain areas with large clusters within the olfactory globes, midbrain, and parts of the hind brain (Figure $5 \mathrm{~A}$ ). Across the whole brain, no gross differences in the expression patterns and distribution of excitatory and inhibitory neurons were observed across all pcdh19 mutant larvae whole brains. However, regional quantification revealed a difference in the number of inhibitory neurons within the tectum (Figure $5 \mathrm{~B}$ ). On average, significantly fewer inhibitory neurons were present in the tectum of $5 \mathrm{dpf} p c d h 19^{+/-}$zebrafish larvae compared to age-matched control larvae or $p c d h 19^{-/-}$ mutants. Comparison of scrambled injected vs. mosaic pcdh19 mutant larvae showed no difference in the average number of inhibitory neurons in the tectum, suggesting that the nonmosaic $p c d h 19^{+/-}$fish display this interneuron phenotype, correlating with the observation that the non-mosaic mutants also have the most pronounced hyperexcitability phenotype. 


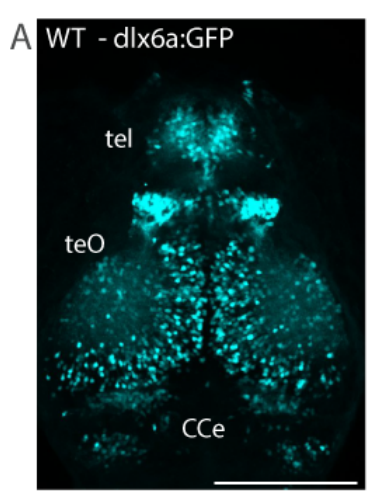

B

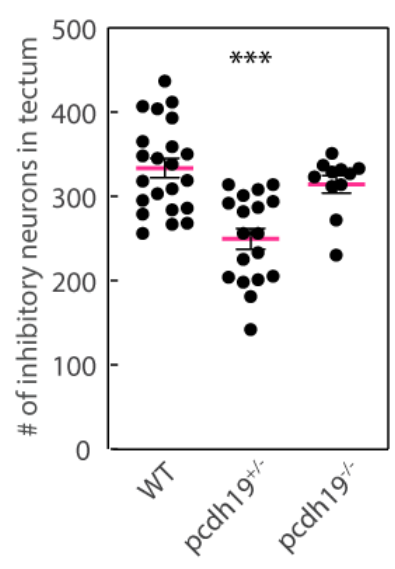

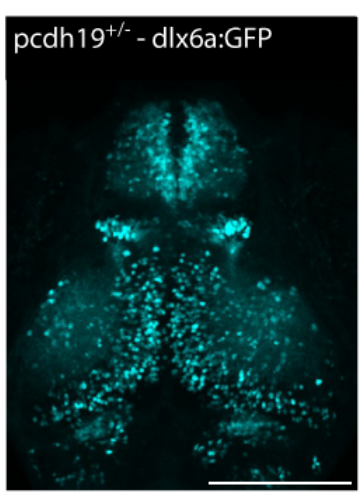
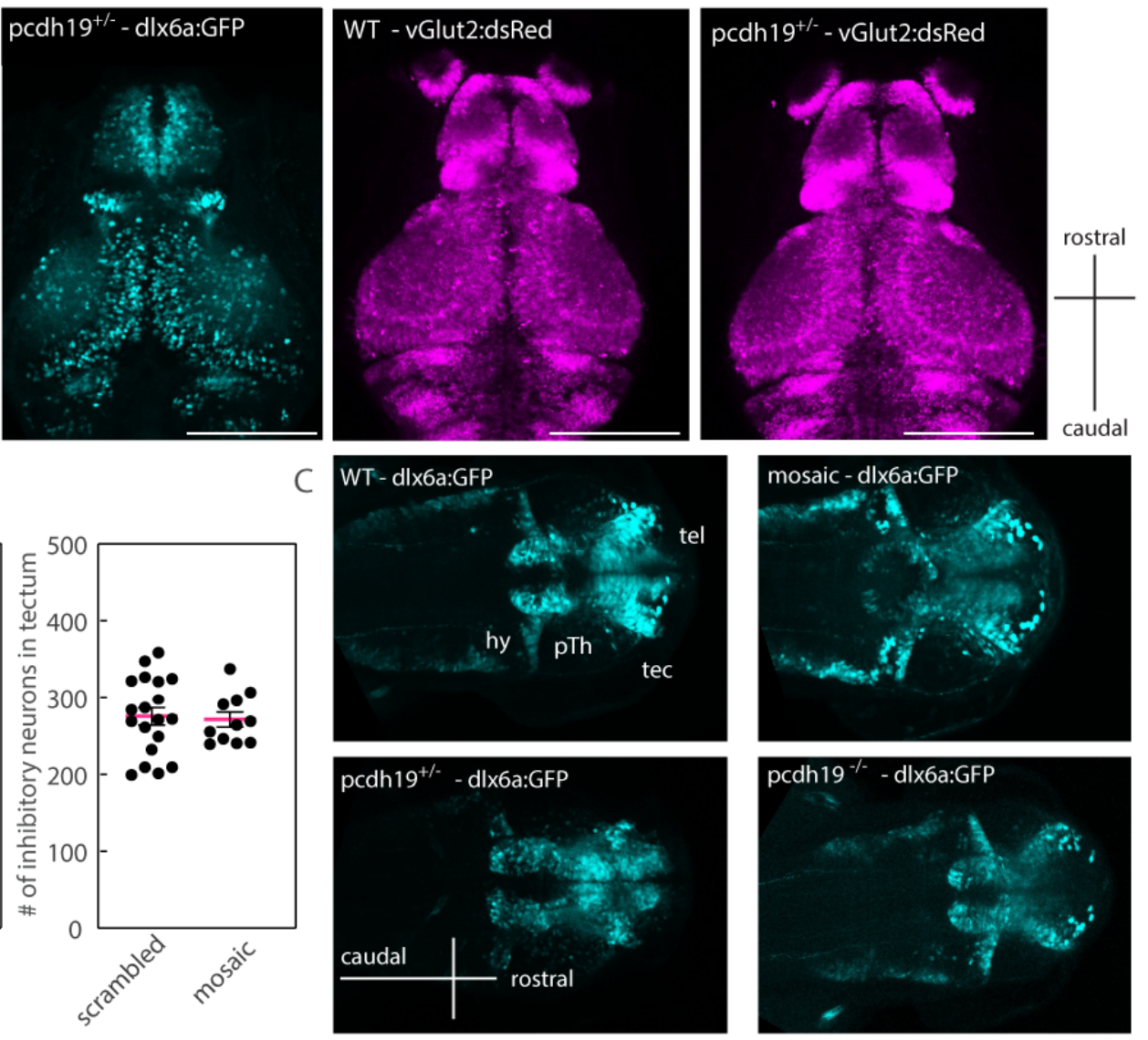

Figure 5: Brain structural abnormalities in $p c d h 19^{+/-}$larvae. (A) Representative confocal maximum intensity projections of a Z-stack taken from a 5dpf WT and a pcdh19+/- mutant crossed with the Tg(dlx6a1.4kbdlx5a/dlx6a:GFP::vglut2:DsRed) line show no gross morphological abnormalities in either the dsRed or the GFP expressing neuron population. Scale bar $200 \mu \mathrm{m}$. (B) Quantification of the number of inhibitory neurons within the tectum shows a reduction only in $p c d h 19^{+/-}$mutants compared to WT controls. $\mathrm{n}=22 \mathrm{WT}, \mathrm{n}=19 \mathrm{pcdh} 19^{+/-}, \mathrm{n}=11 \mathrm{pcdh} 19^{-}$ /-, $n=19$ mosaic, $n=11$ scrambled larvae, one-way ANOVA with Dunnett's multiple comparisons test, $\mathrm{p}<0.0001$. (C) Representative confocal maximum intensity projections of a Z-stack of 2dpf WT, pcdh19+/-, pcdh19//- and mosaic mutants showing an altered, immature neuronal pattern in the $p c d h 19^{+/-}$mutant compared to the WT larvae. tel, telencephalon; teO, tectum opticum; CCe, cerebellar corpus; tec, tectum; pTh, pre-thalamus, hy, hypothalamus.

To determine whether the reduced total number of inhibitory neurons, noted at $5 \mathrm{dpf}$, in these mutants is due to increased cell death, we performed TUNEL assays at 2dpf in pcdh19 KO mutants. Quantification did not show increased cell death in any of the lines, suggesting that destruction of developing interneurons is not the mechanism for the observed interneuron phenotype (Suppl. Fig. 1F). Quantification of the number of inhibitory neurons at 2dpf was impossible since migrating cells form dense clusters and thus, we were unable to identify single GFP-expressing cells at this age. However, we observed differences in the developing brain of $p c d h 19^{+/-}$larvae. While inhibitory neurons in WT embryos start to form distinct clusters in the thalamic brain regions at $2 \mathrm{dpf}$, it appeared that $p c d h 19^{+/-m u t a n t}$ brains had immature patterns of GFP-expressing neurons with overall smaller cell clusters (Figure $5 \mathrm{C}$ ). These are reminiscent of earlier stages of brain development, suggesting transiently delayed development that recovers to normal by 5 dpf. Quantification showed a significantly reduced GFP-fluorescent area in $p c d h 19$ KO larvae compared to WT larvae, indicating either that fewer inhibitory neurons are present, or 
they are more densely clustered at this stage (Suppl. Fig 1G). Together these results suggest that Pcdh19 plays a part in the development of the inhibitory neuronal network and a disruption of Pcdh19 function appears to have a stronger effect in heterozygous pcdh19 KO animals than mosaic mutants.

\section{RNA sequencing reveals abnormal expression patterns of genes involved in brain development.}

We performed RNA sequencing to identify possible molecular mechanisms that might explain the reduced number of inhibitory neurons and the increased excitability revealed by LFP recordings, calcium imaging, and pERK activity. We identified over 1000 differentially expressed genes in non-mosaic pcdh19 KO mutants and more than 5000 in the mosaic mutants with limited overlap between the KO mutants and even less overlap (only 14 differentially expressed genes including pvalb4 and pvalb7) in all three groups (Figure 6B). Since phenotypes were rather mild, we also tested whether pcdh19 loss-of-function resulted in compensatory upregulation of other protocadherins that could take over some of pcdh19's function but we did not observe a consistent upregulation that happened in both groups. As the overlap between the two KO groups was bigger than the overlap with the mosaic mutants, we focused on the KO group for subsequent analyses. In both, the hetero- and homozygous larvae, we observed significant translational downregulation of genes in the following categories, organized by GO terms: eye development, development of the visual system, visual perception, perception of light as well as ATP synthesis and metabolism of ATP, peptides, and amides, among others (Figure 6C). Expression of genes involved in developmental processes, system development, organ-, tissue-, and cell development were upregulated (Figure 6D). Pathway analysis of 894 genes related to disorders associated with hyperexcitability or structural brain developmental abnormalities revealed 46 downregulated and 10 upregulated genes in $p c d h 19^{+/-}$and $p c d h 19^{-/-}$KO larvae with similar expression patterns. These include camkk1b, camk2b1, gpt2, bdnf, grin1a, sv2a, and arhgefgb, gabrd and gabrb2 (Figure $6 \mathrm{E})$. The last three are key components of inhibitory synapse function and thus, interesting candidates that may account for the developmental abnormalities and altered excitation levels. Furthermore, one of the 24 differentially expressed genes that found in all three lines was pvalb7, a gene expressed by inhibitory neurons and orthologous to human PVALB (coding for parvalbumin). Expression patterns of sv2a, arhgef $9 b$, gabrd and gabrb2 were verified in qPCR experiments and showed a reduction in mRNA levels compared to control animals with intact Pcdh19 (Figure 6F). These results suggest that dysregulation of the inhibitory system underlies the hyperexcitable phenotype in pcdh19 mutants and in the setting of PCDH19-related disease. 
A

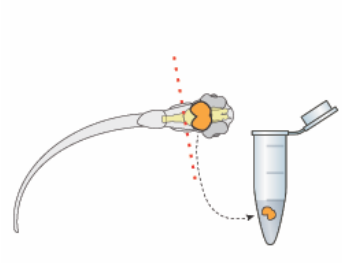

B

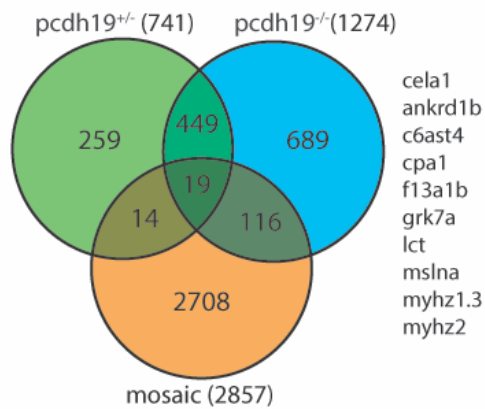

Upregulated
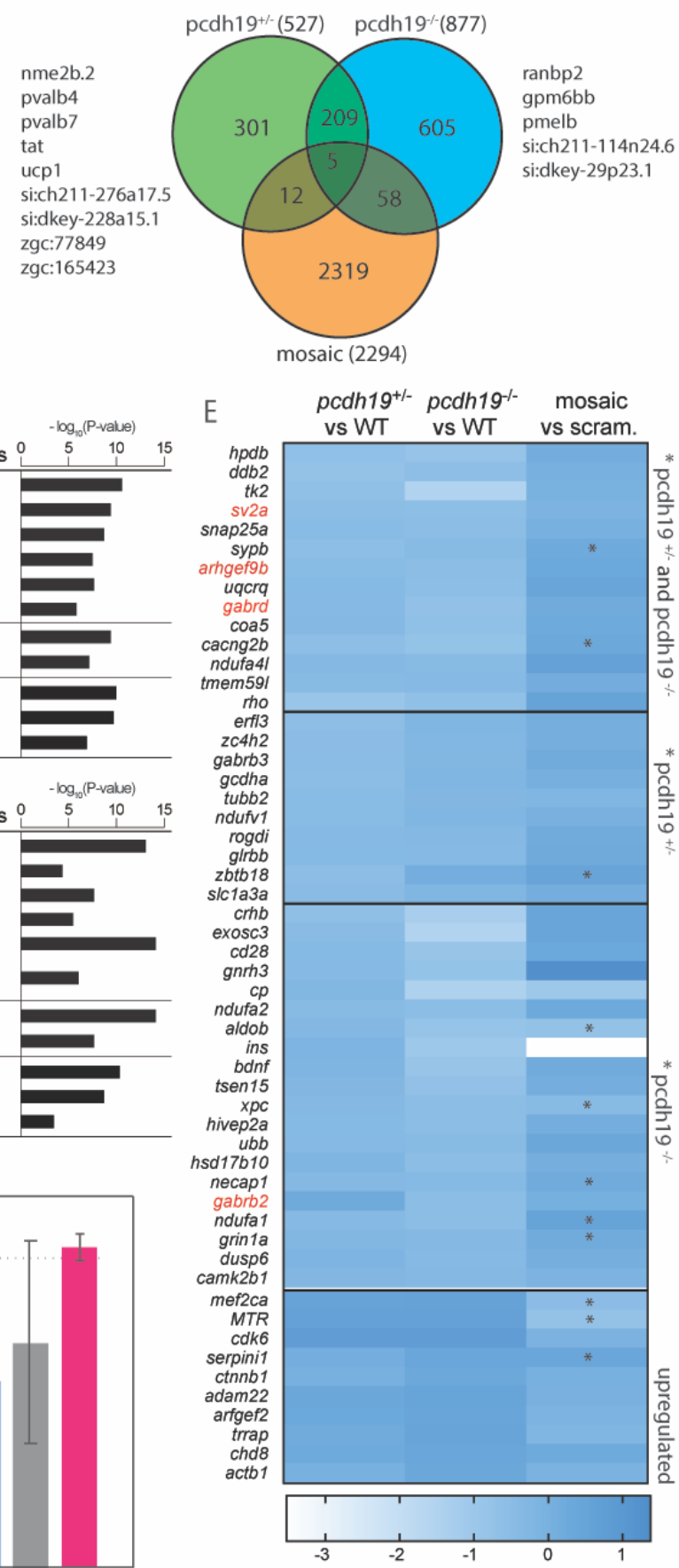

$-\log _{\text {(P) }}$ P-value)

C Functional enrichment in up-regulated genes (pcdh19+- \& pcdh19+- vs WT)

anatomical structure development (GO:0048856) cell development (GO:0048468)

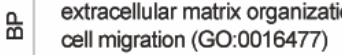

cellular developmental process (GO:0048869)

tissue development (GO:0009888)

u extracellular matrix structural constituent (GO:0005201)

cytoskeletal protein binding (GO:0008092)

focal adhesion (GO:0005925)

$\stackrel{\Perp}{\Sigma}$ cell-substrate junction (GO:0030055) extracellular vesicle (GO:1903561)

D Functional enrichment in down-regulated genes (pcdh19+/ \& pcdh19/vs WT) visual perception (GO:0007601)

photoreceptor cell development (GO:0042461)

protein localization to membrane (GO:0072657)

synaptic signaling (GO:0099536)

peptide biosynthetic process (GO:0043043)

mitochondrial ATP synthesis coupled electron transport (GO:0042775)

U. structural constituent of ribosome (GO:0003735)

structural constituent of eye lens (GO:0005212)

cytosolic large ribosomal subunit (GO:0022625)

neuron projection (GO:0043005)

neuron to neuron synapse (GO:0098984)

\begin{tabular}{|c|c|c|}
\hline & & $-\log _{10}(\mathrm{P}$-value $)$ \\
\hline Fold & $\#$ of genes & $\begin{array}{r}10 \\
1\end{array}$ \\
\hline 1.94 & 85 & 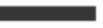 \\
\hline 2.98 & 40 & ש \\
\hline 5.68 & 18 & ש \\
\hline 3.37 & 27 & 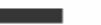 \\
\hline 2.13 & 63 & 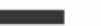 \\
\hline 2.09 & 30 & \\
\hline 9.86 & 14 & \\
\hline 3.04 & 25 & \\
\hline 6.04 & 21 & \\
\hline 5.94 & 21 & \\
\hline 2.39 & 42 & 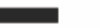 \\
\hline
\end{tabular}

F

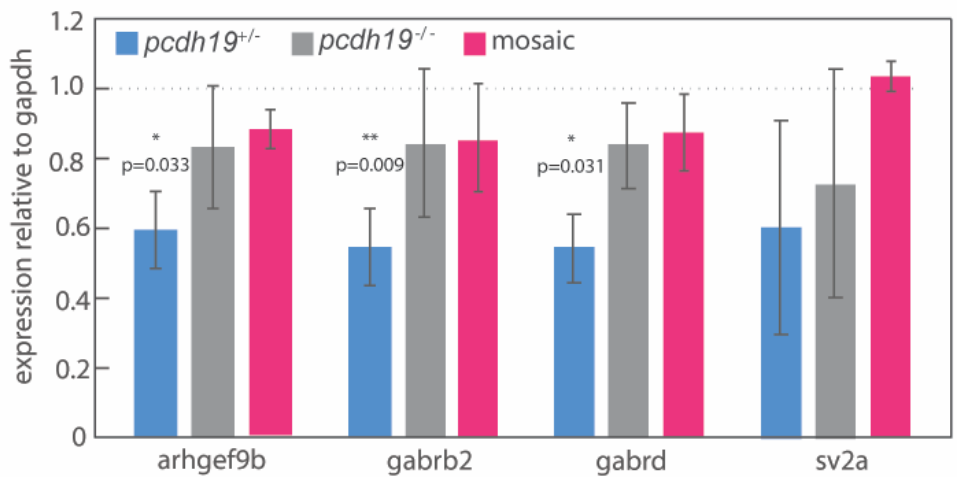

\begin{tabular}{|c|c|c|}
\hline \multirow[b]{2}{*}{ Fold } & \multirow[b]{2}{*}{ \# of genes } & $-\log _{10}(P$-value $)$ \\
\hline & & $\begin{array}{lll}5 & 10 & 15\end{array}$ \\
\hline 7.48 & 23 & \\
\hline 8.79 & 6 & a \\
\hline 3.53 & 27 & \\
\hline 3.16 & 21 & ] \\
\hline 5.67 & 33 & \\
\hline 7.9 & 10 & \\
\hline $\begin{array}{c}9.68 \\
21.39\end{array}$ & 23 & \\
\hline 15.49 & 13 & \\
\hline 2.55 & 50 & 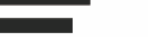 \\
\hline 2.74 & 14 & \\
\hline
\end{tabular}

Figure 6: Transcriptomic analysis of pcdh19 mutants vs. controls. (A) RNA was collected from the heads of 6dpf larvae. (B) Venn diagram illustrating the overlap of differentially expressed genes in $p c d h 19^{+/-}$vs WT, pcdh19-/- vs WT, and mosaic vs scrambled with adjusted p-value <0.05. Numbers indicate the number of genes in each group. Differentially expressed genes present in all three groups are listed on the left of the respective Venn diagram. (C) Functional gene ontology (GO) analysis using genes that are upregulated and downregulated (D) in both $p c d h 19^{+/-}$and pcdh19 ${ }^{-/-}$ mutants. BP, biological process. CC, cellular component. MF, molecular function. (E) A heatmap representation of $\log _{2}$ (fold change) values of epilepsy associated genes that were chosen based on similar expression patterns in $\mathrm{pcdh}^{+/ /-} \mathrm{vs}$ WT, pcdh19/-- vs WT. Mosaic vs scrambled, shown for completeness, most often followed a different expression pattern (stars indicate genes in this group with adjusted $\mathrm{p}$ value <0.05). Genes highlighted in red were chosen for $\mathrm{qRT}$-PCR 
verification. In the first block of downregulated genes adjusted $\mathrm{p}$ value $<0.05$ in both groups, second block adjusted $\mathrm{p}$ value $<0.05$ in the $p c d h 19^{+/-}$only, third block adjusted $\mathrm{p}$ values $<0.05$ in $p c d h 19^{-/-}$only, last block shows upregulated genes with adjusted p value <0.05. (F) Average expression levels, relative to gapdh of the selected, down-regulated genes confirms reduced arhgefgb, gabrb2, and gabrd transcript levels compared to WT expression, which is normalized to 1 . $\mathrm{n}=9 \mathrm{WT}, \mathrm{n}=9$ pcdh1 $19^{+/-}, \mathrm{n}=6 \mathrm{pcdh1} 19^{-/-}, \mathrm{n}=4$ scrambled, $\mathrm{n}=4$ mosaic head pools, one-way ANOVA with Tukey's multiple comparisons test.

\section{Mutant pcdh19 zebrafish do not display evidence of anxiety or impaired spatial learning.}

Because patients with $\mathrm{PCDH19}$ variants often display neuropsychiatric comorbidities with intellectual disability and/or autism, behavioral dysregulation, and obsessive features (Samanta, 2020), we evaluated our $p c d h 19$ zebrafish mutants for learning defects and features of anxiety, which have established correlates in zebrafish. We found no difference between mutant and control larvae using the thigmotaxis, or wall-hugging, assay for larval zebrafish, which is considered a correlate for anxiety (Schnorr et al., 2012). WT and scrambled injected larvae spend on average $71.8 \%$ and $70.9 \%$ of the time 'hugging' the wall, while this proportion is increased to 88.5\% in WT larvae treated with the anxiogenic caffeine, indicative for increased anxiety levels $(50 \mathrm{mg} / \mathrm{L})$ (Figure $7 \mathrm{~A}$ and $\mathrm{B})$. We did not observe a statistically significant difference in the wallhugging behavior of $p c d h 19^{-/-} \mathrm{KO}$ or mosaic mutants compared to their respective controls, and we observed a significant reduction of thigmotaxis in $p c d h 19^{+/-}$fish. As neuropsychiatric dysfunction tends to exacerbate with age in human PCDH19-CE, we also looked at older pcdh19 mutant fish. We found weak evidence for anxiety-like behavior only in pcdh19 mosaic mutants in the bottom dwelling test using adult zebrafish (Figure $7 \mathrm{C}, \mathrm{D}, \mathrm{E}$ ). While WT fish spent on average $93 \pm 61 \mathrm{sec}$ in total in the top compartment of the test tank, $p c d h 19^{+/-}$and $p c d h 19^{-/-}$fish tend to spend more of time in the top $(127 \pm 85,151 \pm 56 \mathrm{sec}$ respectively), indicating no anxiety-like behavior. Scrambled and mosaic injected adults spent even more time in the top compartment but to a similar degree (Figure 7D). Interestingly, the mosaic pcdh19 mutants had significantly more zone transitions compared to the scrambled injected and pcdh19 KO fish (Figure $7 \mathrm{E}$ ), a behavior that is consistent with anxiety-like behavior. However, this group was also the only group in which almost all of tested animals entered the top compartment at least once, while in all other groups a substantial proportion of fish remained motionless at the bottom of the tank (Figure $7 \mathrm{~F}$ ). Based on these findings, we conclude that pcdh19 KO mutant zebrafish do not display a strikingly increased anxiety-like phenotype, while pcdh19 mosaic mutants show tendencies towards an anxiety-related phenotype. 
a) WT

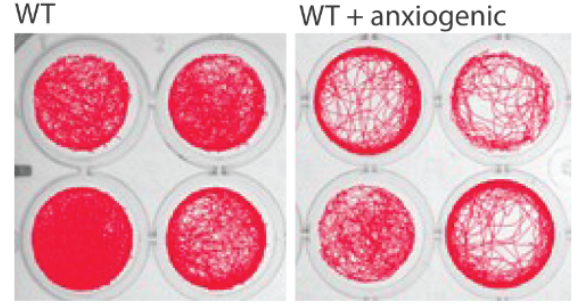

c)

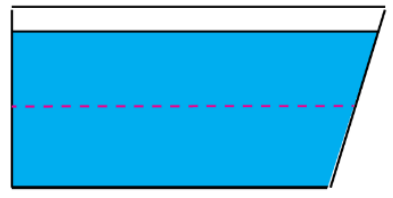

g)

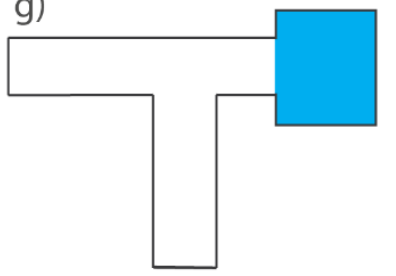

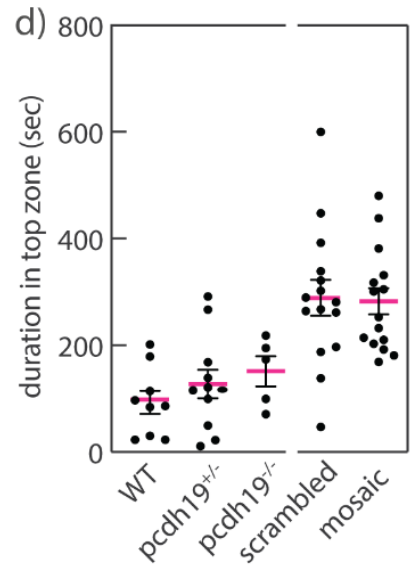
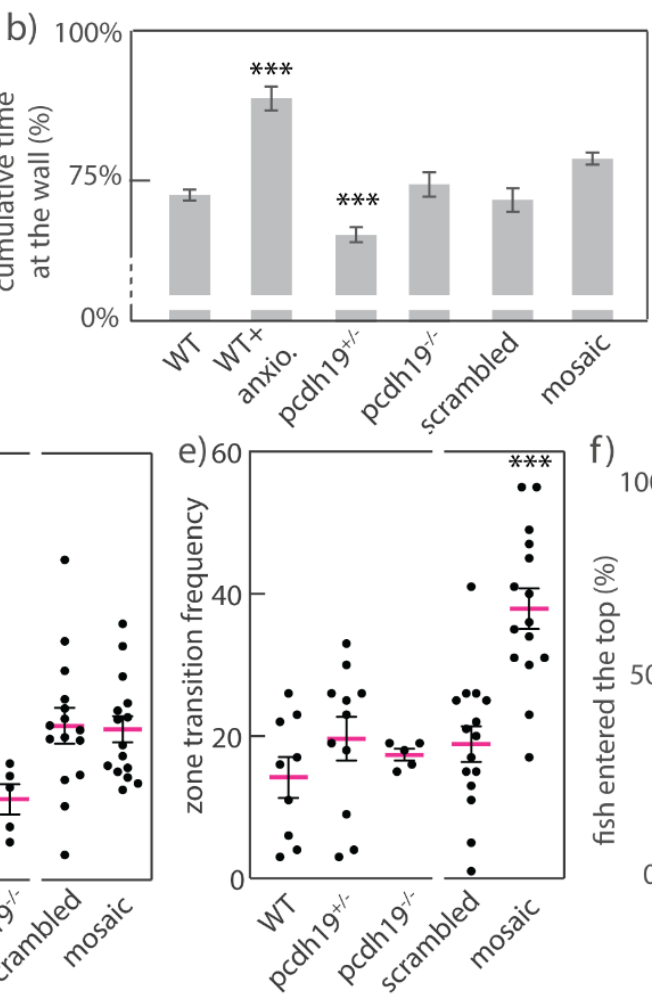

f)
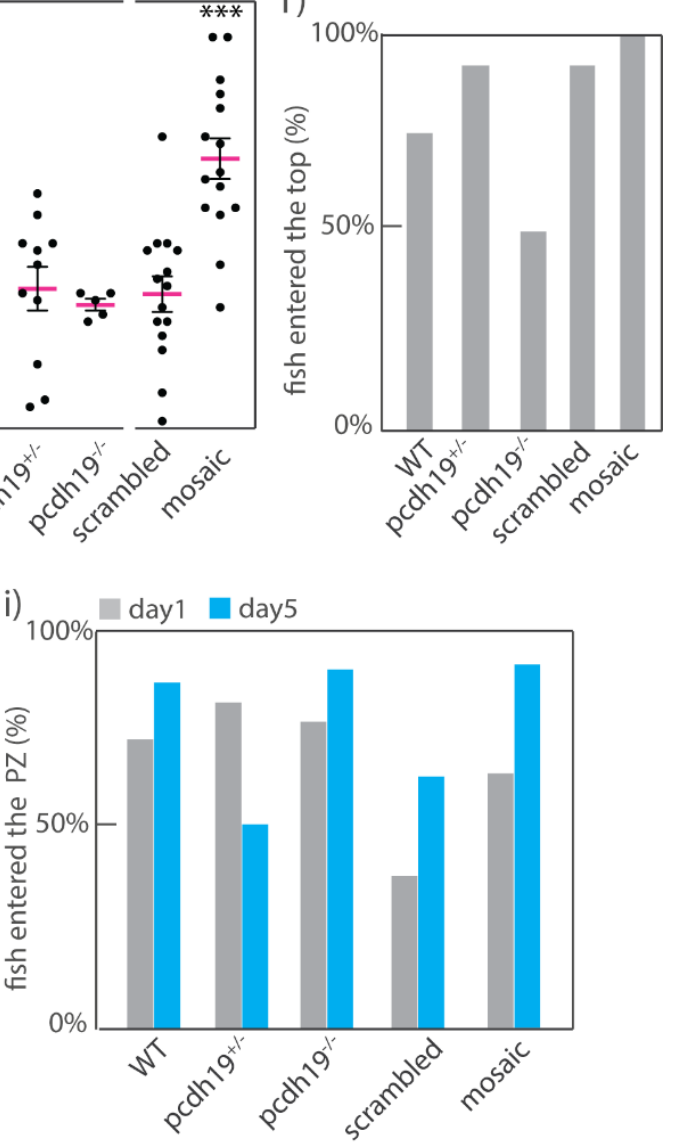

Figure 7: Behavioral paradigms to assess for features of anxiety and learning defects in zebrafish. (A) Movement plots of 4 representative WT larvae untreated and treated with the anxiogenic caffeine in a 24-well plate over 30 min. (B) Quantification of the time spent at the wall shows only larvae treated with the anxiogenic spend more time at the borders of the well, while $p c d h 19^{+/-}$larvae spent significantly less time close to the wall. $n=367$ WT, $n=311 p c d h 19^{+/-}$, $\mathrm{n}=91$ pcdh1 $1 \mathrm{~g}^{-/}, \mathrm{n}=283$ mosaic, $\mathrm{n}=60$ scrambled, $\mathrm{n}=59 \mathrm{WT}+$ caffeine treated leave, one-way ANOVA with Tukey's multiple comparisons test, ${ }^{* * *} \mathrm{p}<0.001$. (C) Schematic of the bottom-dwelling test tank. (D) Quantification of the duration the adult zebrafish spent in the top compartment of the test tank, that is unchanged between control and pcdh19 mutant animals. (E) The frequency of transitions between bottom and top compartment is significantly increased in mosaic pcdh19 mutants compared to their controls. (F) The percentage of fish that entered the top compartment is lower for WT and pcdh19-/- fish compared to the other groups. $\mathrm{n}=12 \mathrm{WT}, \mathrm{n}=12$ pcdh $19^{+/-}, \mathrm{n}=10$ pcdh1 $9^{-}$ /-, $\mathrm{n}=15$ mosaic, $\mathrm{n}=15$ scrambled fish, one-way ANOVA with Sidak's multiple comparisons test, $\mathrm{p}<0.0001$. (G) Schematic of the T-maze tank. The blue compartment depicts the preference zone (PZ). (H) Average latency to enter the $\mathrm{PZ}$ on the 5 consecutive training days is similar for all compared groups. (I) The percentage of fish that entered the $\mathrm{PZ}$ on day1 or 5 varies between groups. $\mathrm{n}=36 \mathrm{WT}, \mathrm{n}=28$ pcdh1 $9^{+/-}, \mathrm{n}=36 \mathrm{pcdh} 19^{-/-}, \mathrm{n}=64$ mosaic, $\mathrm{n}=16$ scrambled fish, one-way ANOVA with Dunnett`s multiple comparisons test. 
Using the T-maze test with adult zebrafish (Figure $7 \mathrm{G}$ ), we found no defects in the learning capabilities of pcdh19 mutants. Both, the performance of mutant and the WT increased similarly over the duration of the trial. The average latency for WT zebrafish to reach the preference zone (PZ) went down from $93.7 \pm 76$ s on day 1 to $23.5 \pm 41$ s on day 5 , this was a 4 fold and statistically significant improvement in reaching the preference zone (Figure $7 \mathrm{H}$ ). Comparably, all mutant pcdh19 zebrafish improved significantly from day1 to day5 with mosaic and $p c d h 19^{+/-}$animals showing the most dramatic response (with 6.4- and 4.1-fold improvement respectively), pcdh19/- performance improved the least (2.7-fold improvement relative to day 1). Comparing latency to reach the preference zone on day 1 or on day 5 between WT and pcdh19 mutants did not show any significant differences (Figure $7 \mathrm{H}$ ). However, a considerable number of fish were excluded from the analysis because they either failed to move during the $5 \mathrm{~min}$ recording period or failed to reach the preference zone (Figure $7 \mathrm{I}$ ). Together, these results show that both, mosaic and non-mosaic pcdh19 mutants did not show overt signs of increased anxiety or cognitive decline compared to age-matched control animals, indicating that distinct Pcdh19 expression patterns have no severe impact on learning and memory or anxiety in 4- to 5-month-old zebrafish.

\section{Discussion}

A prevailing hypothesis is that human $\mathrm{PCDH} 19$-associated disease phenotypes result from the presence of cellular mosaicism in the brain, with clusters of neurons expressing WT and others expressing mutant PCDH19 protein. To test this hypothesis, we assayed both mosaic and nonmosaic pcdh19 loss-of-function zebrafish larvae for features related to epilepsy and comorbid features associated with human disease, as well as for underlying neurodevelopmental abnormalities that might be responsible for these features. Our most striking finding was abnormal hyperexcitability in mosaic and non-mosaic loss-of-function zebrafish larvae, as evidenced by spontaneous epileptiform discharges observed by tectal electrophysiological recording. This is a feature associated with epilepsy previously shown in a zebrafish model for Dravet syndrome (Baraban et al., 2013). These findings were confirmed by using multiple pcdh19 loss-of-function lines and by acutely injecting a different sgRNA to target pcdh19 at a distinct genomic position. Surprisingly, we saw only limited indications of abnormal excitability using GCaMP-mediated calcium imaging and behavioral based locomotor activity paradigms both at baseline and during provoking conditions using light, heat and the proconvulsant PTZ at different concentrations, all established methods to assay hyperexcitable states in model organisms (Afrikanova et al., 2013; Hortopan et al., 2010; Hunt et al., 2012; Liu and Baraban, 2019). Using these assays, the strongest hyperexcitability phenotype was observed in non-mosaic, heterozygous pcdh19 mutant larvae showing spontaneous and PTZ-induced behavioral seizures as well as abnormal calcium events. We conclude that the nature of the pathology, and its resultant hyperexcitability, generated by mosaic and non-mosaic pcdh19 LOF mutations in zebrafish larvae requires a sensitive detection method (tectal LFP recordings) to consistently identify evidence of hyperexcitability in all lines. Indeed, the failure to detect seizure-like events using behavioral assays in the setting of a hyperexcitable neuronal network is not an uncommon phenomenon, as recently demonstrated in a large zebrafish epilepsy screen (Griffin et al., 2021).

Relative changes in pERK antibody fluorescence, employed to provide neuroanatomical localization of neuronal activity (Thyme et al., 2019), showed complex results in our mosaic and non-mosaic pcdh19 mutants. Mosaic mutants showed marked changes in neuronal activity in a 
widespread pattern which partly overlaps with tectum, but heterozygous pcdh19//- mutants-the same genotype that showed evidence of hyperexcitability by other modalities-showed more subtle abnormalities using pERK fluorescence. Since this method only visualizes changes in neuronal activity that occurred approximately 10min prior to preparation, it may not be ideally suited to image short, transient events such as seizures. The abnormal pERK patterns found in the mosaic mutants may therefore represent longer lasting, persistent changes in neuronal activity patterns that are different form the non-mosaic pcdh19 lines and may not represent the presence of seizures as such but rather a baseline, tonic hyperexcitable state.

In order to further explain the hyperexcitability pcdh19 mutant phenotype, we assessed neuronal numbers and performed transcriptional profiling. We observed loss of inhibitory neurons at 2dpf and $5 \mathrm{dpf}$ with normal TUNEL staining and reduced transcripts of key genes involved in inhibitory synapse function and neuronal development in heterozygous loss-of-function mutants. These observations collectively represent changes consistent with a hyperexcitable neuronal network. Our results suggest a failure of development of inhibitory neurons in stable non-mosaic pcdh19 mutants that is not observed in mosaic loss-of-function mutants. Transcripts for arhgefgb (encoding gephyrin, a protein stabilizing post-synaptic receptor proteins at inhibitory synapses) and $g a b r b 2 a$ and $g a b r d$ (GABAa receptor subunits) were significantly reduced in $p c d h 19^{+/-}$larvae and only slightly reduced in $p c d h 19^{-/-}$larvae, which may explain the more severe hyperexcitability phenotype present in heterozygous pcdh19 mutants. Consistent with our findings, experiments silencing Pcdh19 in cultured rat neurons showed reduced GABAaR and GAD65/67-positive puncta and reduced mIPSC frequency with slower decay kinetics, suggesting a Pcdh19- mediated mechanism is necessary for the composition and function of the inhibitory synapse (Bassani et al., 2018). With regard to transcriptional profiling, we were surprised that the altered gene expression in these fish showed mostly non-overlapping profiles between the mosaic and nonmosaic mutants which suggest large differences in the downstream consequences, but with some commonalities centered on inhibitory neuron function. Given the similar abnormal epileptiform discharges across these models, this suggests either that the transcriptional changes are related to the mosaic vs non-mosaic patterns of LOF or arise from the different kind of manipulation (acute injections vs. KO lines), the use of aggregate tissue or the pathophysiological consequences are not fully reflected in the downstream gene changes. It is possible that if the function of Pcdh19 is most critical in certain neuronal subtypes, such as the inhibitory neurons, that the transcriptional changes in these specific subtypes are not sensitively detected by this approach.

Common neuropsychiatric features of the human PCDH19-related disorder include intellectual disability, autism, and obsessive-compulsive disorder which tend to worsen over time (Samanta, 2020). We therefore evaluated not only larval but also adult zebrafish for these abnormalities. Similar to published reports in mice that assessed changes in anxiety and memory-related behavior (Hayashi et al., 2017; Pederick et al., 2016), none of the adult fish displayed consistent or striking abnormalities in behavior using two different tests that assess learning and memory performance and anxiety-like states. The discrepancy between the severe neuropsychiatric comorbidities in humans and the rather mild reported phenotypes in mice and zebrafish, if any, suggests a human-specific function of PCDH19 in the brain or a lack of more adequate assays for complex behaviors in the animal models. 
The extent to which non-mosaic Pcdh19 loss-of-function contributes to abnormal hyperexcitability and epilepsy remains unresolved. As the prevailing theory postulates that cellular interference in the setting of mosaic LOF is required for a phenotype (Pederick et al., 2018), we conclude that alternative mechanisms must play a role to account for our observations that are consistent with hyperexcitability in non-mosaic heterozygous or homozygous pcdh19 mutant zebrafish. This hypothesis is supported by the presence of behavioral abnormalities in human male carriers (unpublished observations), abnormalities in neuronal network development and function in the optic tectum of Pcdh19 mutant zebrafish (Light et al 2019; Cooper 2015), and abnormalities in pcdh19 KO mice. This study showed heightened evoked seizure susceptibility in both female $P c d h 19^{+/-}$(mosaic) and female $P c d h 19^{-/-}$(non-mosaic) mice compared to WT and male hemizygous mice (Rakotomamonjy et al., 2020), strengthening the hypothesis that mosaicism-independent mechanisms also contribute to PCDH19 disease phenotypes.

The differences seen across different zebrafish pcdh19 mutant lines are reminiscent of differences observed in studies of other model systems, including mouse models of epilepsy (Wang and Frankel, 2021). Given that the protocadherin gene family is well conserved across species, and that the zebrafish pcdh19 gene shares 73\% homology with human PCDH19 (Blevins et al., 2011; $\mathrm{Wu}, 2005$ ), the zebrafish systems allows for robust evaluation of phenotypes that correlate with human patients with pathogenic variants in PCDH19. Indeed, zebrafish models of other genetic epilepsies have been well described and even used successfully in drug screens (Baraban et al., 2013; Eimon et al., 2018; Grone et al., 2016). The lack of spontaneous behavioral seizures in the mosaic or non-mosaic Pcdh19 loss-of-function fish, despite abnormal electrophysiological findings, does not diminish the significance of the positive findings observed and is consistent with several previously reported models of genes responsible for human epilepsy. A recent large scale epilepsy screen reported a low prevalence of behavioral seizures in a set of 40 zebrafish lines with variants in catastrophic childhood epilepsy genes as well as surprisingly low prevalence of epileptiform abnormalities in electrophysiological recordings (Griffin et al., 2021). A caveat to our study is that due to technical limitations, we could not test for the presence of segregated and abnormally clustered cell populations in the brain in the mosaic mutants. Furthermore, zebrafish larvae are not sexually dimorphic and thus, this disease model does not allow for the evaluation of sex-dependent differences. Given that the most consistent phenotypic abnormality relied on electrophysiological experiments, we advocate for including such experiments, at least as validation experiments, in drug screens for the identification of novel therapeutic compounds, which are readily feasible in zebrafish larvae (Griffin, 2021).

In summary, we demonstrate that mosaic and non-mosaic pcdh19 mutant zebrafish recapitulate several features of human PCDH19-CE, including the core feature of brain hyperexcitability in the zebrafish models that correlates with the network dysfunction resulting in epilepsy in human patients. We provide evidence suggesting that the mechanism for hyperexcitability in non-mosaic Pcdh19 mutants may involve abnormal inhibitory neuron development and may partly overlap with the pathophysiology of mosaic pcdh19 loss-of-function. Understanding the molecular basis of brain dysfunction that arises from mosaic and non-mosaic mutant Pcdh19 expression in zebrafish provides a fundamental step towards understanding PCDH19-CE disease mechanisms, fostering future studies to develop mechanism-based strategies to treat the seizures and perhaps the other neurodevelopmental features of PCDH19-CE. Specifically, the identification of a 
reduction of inhibitory neurons during development and aberrant GABA subunit expression suggest that modifying GABAergic activity, which can be achieved pharmacologically in patients with some classes of anti-seizure medications (e.g., benzodiazepines, barbiturates), may represent a more effective treatment mechanism than many of the other mechanisms of conventional antiseizure medications (e.g., sodium channel blockers).

\section{Methods}

Zebrafish Maintenance

All zebrafish experiments were approved and conducted in accordance with the standards of the Boston Children's Hospital (BCH) Institutional Animal Care and Use Committee (IACUC). Fish were maintained at $79^{\circ} \mathrm{F}$ on a $10 \mathrm{~h}-14 \mathrm{~h}$ dark-light cycle in a dedicated zebrafish core facility. All experiments were performed in Danio rerio strain $\mathrm{AB}$ or TAB.

\section{Guide RNA design and microinjections}

Single guide RNAs (sgRNAs) were designed using the CHOPCHOP online tool v1 (https://chopchop.rc.fas.harvard.edu) and selected using its ranking algorithm based on target specificity and minimization of off-target effects. We used pcdh19 sgRNAs targeting the beginning of exon 1 and exon 2 to maximize loss-of-function of the protein, based on our observation that most patient mutations are located in exon 1, with a few in exon 2, and almost none in the remaining exons. Microinjections into embryos at the one-cell stage were performed as described previously (Dentici et al., 2021) with a mixture of $1 \mu \mathrm{l}$ synthetic guide RNAs (pcdh19 oligo 2/exon1: GGTGTATTTCAAATTGAACA or pcdh19 oligo 3/exon 2: GGAGACGGACAAGATGAATG at 200ong/ $\mu$ l, Synthego, Redwood City, CA, USA) and $1 \mu$ l recombinant Cas9 protein $(1 \mu \mathrm{g} / \mu \mathrm{l}$, PNA Bio, Thousand Oaks, CA, USA) and $2 \mu$ RNAse-free water. For control experiments, a scrambled sgRNA (ACAAGGAGGTAGGCGAGAAC) that does not target zebrafish DNA was injected. A total of 2 nl mix per embryo was injected using clipped glass capillaries and a microinjector.

Genotyping and PCR

To determine sgRNA efficiency and to confirm the different genotypes in pcdh19 KO fish, fin clips were cut from adult 3-month-old fish and DNA extracted using the HotShot protocol (Meeker et al., 2007). Euthanized larvae were dissolved in $5 \mathrm{O} \mu \mathrm{l} 5 \mathrm{OmM} \mathrm{NaOH}$ by heating for $20 \mathrm{~min}$ to $95^{\circ} \mathrm{C}$ followed by addition of $5 \mu \mathrm{l} 1 \mathrm{M}$ Tris solution. Subsequent PCR using 2xPlatinum Taq polymerase and Sanger sequencing was performed with primers summarized in Table 1 . The amount of indels in pcdh19 mosaic larvae and the potentially resulting knockdown percentage was determined via Inference of CRISPR Edits analysis (https://www.synthego.com/products/bioinformatics/crispr-analysis).

Assessment for spontaneous and induced seizures

At 6dpf larvae with pcdh19 mutations were transferred into wells (one each) of a 96-well plate, placed into the DanioVision video-tracking system (Noldus, Netherlands), and tracked with the Ethovision software after a 20-minute acclimation period. For 6omin, baseline swim patterns were recorded and evaluated for seizure-like episodes. PTZ protocol: Larvae were subjected to a 3omin exposure to 2 different concentrations of PTZ (0.1mM and 2.5mM). Hyperthermia protocol: Individual 6dpf larvae were transferred to a 96-well plate submerged in room temperature $\left(22^{\circ} \mathrm{C}\right)$ water. Baseline swim patterns were recorded for 2omin. Then, the water 
temperature was slowly elevated over the course of $20 \mathrm{~min}$ from room temperature to $37^{\circ} \mathrm{C}$, and swim patterns were recorded. Afterwards, swim behavior was assessed for seizure-like swim patterns and compared to parameters during the baseline period. As a control for the KO fish lines we used WT animals, and for the mosaic fish we used scrambled gRNA injected animals.

For the analysis, parameters including total distance moved and maximal velocity for each fish were measured in 5 -second time bins. Based on these parameters, an automated seizure detection paradigm was developed and used to identify the presence of seizure-like activity in each animal per time bin (Lin et al., 2018).

\section{$\underline{\text { Behavioral tests }}$}

T-maze cognition test. We designed an asymmetrical T-maze that has one bigger compartment on the right arm versus the left arm. The bigger compartment represents the preference zone (PZ) which is more favorable since its bigger and enriched, which fish are expected to seek out and stay in (Darland and Dowling, 2001). Adult fish (4-5 months old) were released to the maze and are allowed to explore for $5 \mathrm{~min}$ once a day for 5 consecutive days. The latency from entering the maze to reaching the preference zone as well as the cumulative time the fish spend in the preference zone were analyzed for each fish. Fish that did not move during the whole trial or that did not reach the preference zone were excluded from the analysis. The number of fish that met these exclusion criteria are reported for each genotype.

Bottom dwelling anxiety test. Single, adult, naive fish were placed in a rectangular shaped tank which was virtually divided into upper and lower halves. Their swim behavior was recorded immediately after placement into the new tank for 10min. When exposed to a new environment fish initially seek out the darker, "protective" bottom of a tank to avoid detection by predators and subsequent vertical exploration behavior (Kysil et al., 2017). The higher the anxiety score, the longer the fish stay in the bottom compartment or the more often they will return to the bottom after short episodes of exploring the top compartment. Time spent at the top and bottom-to-top transitions were assessed for each fish.

Transcriptional analysis.

RNA was isolated from heads of 6dpf fish. Each head was individually stored in RNAlater (Invitrogen, USA), and the corresponding tail was used for DNA extraction and Sanger sequencing to confirm genotype. For RNA isolation the tissue from 25 Sanger-confirmed larvae was pooled (to achieve the minimum required RNA concentration), and RNA was then extracted with the QIAGEN RNA isolation kit according to the manufacturers guide. RNA sequencing with differential gene expression analysis was performed at Novogene. For qPCR, 10oug of RNA was reverse transcribed into cDNA using the SuperScript III (Invitrogen, USA) kit according to manufacturer's instructions. qPCR was performed on a QuantStudio 3 -96-Well 0.1-mL Block and the SYBR green PowerUp kit (Applied Biosystems, USA) according to manufacturer's instructions. Standard curves were obtained for each used primer pair (Table 1), and efficiencies were above 1.8 for each combination. Transcript levels were calculated by the $\Delta \Delta$ ct method (Livak and Schmittgen, 2001). Gene ontology (GO) analysis was done with differentially expressed genes using (http://geneontology.org) (Ashburner et al., 2000) only including genes with a p-values of less than $10^{-3}$. The false discovery rate (FDR) was calculated by the Benjamini-Hochberg method. 
The 894 epilepsy genes used for pathway analysis were a collection of genes found in the literature.

Table 1: primers used in PCR and qPCR

\begin{tabular}{|l|l|l|}
\hline gene & Forward primer & Reverse primer \\
\hline pcdh19 exon 1 & TGCCAGACTGTGATTTTGTTCG & TGCTTGGTGATGAGCAGTCC \\
\hline$p c d h 19$ exon 2 & CGTGCCGCCAATGAATATGG & AAGGTGTGGTGGTAGCCATG \\
\hline elfa & CTTCTCAGGCTGACTGTGC & CCGCTAGCATTACCCTCC \\
\hline gapdh & GTGGAGTCTACTGGTGTCTTC & GTGCAGGAGGCATTGCTTACA \\
\hline sv2a & ACTACTGCCTTCGGTTTTCTG & TATTGAAGCACCTGACCACGA \\
\hline gabrb2 & GACTCCCGATGATATCCGG & TCTGGGTAGCGTGGCGC \\
\hline gabrd & CGATGGTGAAGAATGGAAAGC & AAGACGGCACGAGCGTAGA \\
\hline arhgefgb & AAGATGGTTTCTGGATCTATTCC & ATGCTCCTGTGCCGTGTAC \\
\hline
\end{tabular}

\section{$\underline{\text { LFP recordings }}$}

Electrophysiological recordings were obtained from larval zebrafish using previously described methods (Baraban et al., 2013). Larval zebrafish (6 to $7 \mathrm{dpf}$ ) were paralyzed with alphabungarotoxin $2 \mathrm{mg} / \mathrm{ml}$ (Invitrogen, USA), then embedded dorsal side up in $50 \mu \mathrm{l}$ of $1.2 \%$ lowmelting point agarose (Fisher Scientific, USA) on a recording chamber. The chamber was placed under a Nikon Eclipse FN1 microscope and perfused with zebrafish recording solution containing: $117 \mathrm{mM} \mathrm{NaCl}_{4} .7 \mathrm{mM} \mathrm{KCl}, 2.5 \mathrm{mM} \mathrm{NaHCO}_{3}, 2.5 \mathrm{mM} \mathrm{CaCl}_{2}, 1.2 \mathrm{mM} \mathrm{MgCl}_{2}, 1.2 \mathrm{mM} \mathrm{NaH}_{2} \mathrm{PO}_{4}, 11 \mathrm{mM}$ glucose (Sigma-Aldrich, USA) dissolved in distilled water, brought to a $\mathrm{pH}$ of 7.4, and vacuumfiltered through a $0.22 \mu \mathrm{m}$ filter. The specimen was perfused with warm $\left(28.5^{\circ} \mathrm{C}\right)$ recording solution at a rate of $1-2 \mathrm{ml} / \mathrm{min}$ throughout the recording. A borosilicate glass electrode (1-7M $\Omega$ resistance) filled with $2 \mathrm{M} \mathrm{NaCl}$ was placed in the optic tectum using a micromanipulator with visual guidance under the light microscope. Local field potential (LFP) recordings were collected using Clampex software (Molecular Devices, USA). All recordings were performed in currentclamp mode, low-pass filtered at $1 \mathrm{~Hz}$, high-pass filtered at $1 \mathrm{kHz}$, amplified at a gain of $500 \mathrm{x}$, and sampled at 10kHz (Axopatch 200B amplifier, Digidata 1440A digitizer, Molecular Devices, USA). After a baseline recording of 50min, PTZ (pentylenetetrazole) 40mM (dissolved in recording solution) was added to the recording chamber. The recording continued for 2omin after the addition of PTZ. Fish were observed under the microscope frequently throughout the experiment for presence of heartbeat and circulation in the head and absence of movement. Only recordings with a visible heartbeat throughout were included in the analysis.

Analysis of LFP data

All electrophysiology was analyzed with a custom MATLAB software framework developed by an investigator blind to status of the experiment. The framework first passes the raw data through a filtering stage - based on the Savitzky-Golay filtering algorithm. The software uses the filtered voltages to automatically detect the measurement noise floor by fitting the core of the histogram of the filtered voltages with a Gaussian distribution. This noise floor (0.006-0.012mV) allows for a robust detection of all spikes. These spikes are further categorized into alpha and beta events based on their signal amplitude. This distinction into alpha and beta event can be directly obtained from a histogram of all detected spike amplitudes in which we observe two distinct 
features: a narrow peak with low signal amplitude - referred to as alpha events - and a second broad peak with large signal amplitude - referred to as beta events. The threshold discriminating between alpha and beta events is 0.08-0.12mV depending on the noise (after completion of the manuscript, the authors became aware of a related classification (Griffin, 2021)). A final stage of the software groups the detected alpha and beta events into bursts in case the following event occurs within a 1 second time window.

\section{Calcium imaging}

Pcdh19 mutant zebrafish were crossed with Tg(elav3:GCaMP6s); mitfanac/nac (abbreviated, GCaMP6) zebrafish in the nacre background to achieve GCaMP6-expressing, transparent pcdh19 mutant lines. Heterozygous pcdh19 mutants were bred for Ca2+ fluorescence detection at $6 \mathrm{dpf}$. To this end, individual unrestrained larvae were placed into a well of a 96-well optical plate in $100 \mu$ fish water compatible with the Hamamatsu FDSS70ooEX fluorescent plate reader. The fish were not evaluated for 2omin to allow time for them to acclimate to the new environment. Specimens were illuminated from above via Xenon lamp passed through a 480 mm filter. Epifluorescence from below the specimen is passed through a 540nm filter and collected by CCD, allowing all wells to be recorded simultaneously. Data is sampled at approximately $12.7 \mathrm{~Hz}$ (79msec interval), 16-bit pixel depth and 2x2 binning. Fish were recorded for 30min untreated, 30min treated with $1 \mathrm{mM}$ and 30min treated with 10mM PTZ. Afterwards heartbeat is assessed and DNA is extracted for genotype determination. Analysis is performed in MATLAB to extract position, linear and angular velocity, and changes in calcium fluorescence using a moving average $\Delta \mathrm{F} / \mathrm{F}_{\mathrm{o}}$ method.

For high-resolution Calcium imaging, living GCaMP6 larvae (5-7dpf) were immobilized with $5 \mathrm{mM}$ tubocurare and embedded in $1.2 \%$ agarose for imaging using a Zeiss Lightsheet.Z1 microscope equipped with $20 \mathrm{X} / 1$.o NA objective. Calcium fluorescence was stimulated by two coplanar light sheets positioned at 90 degrees from the detection axis using 488nm laser. To assess abnormalities in spontaneous brain activity, two paradigms were used: A) whole brain activity, encompassing $\sim 13$ slices with 22uM slice interval at a rate of 1.8seconds per stack; B) z-section through optic tectum at $10 \mathrm{~Hz}$. Samples were immersed in temperature-controlled fish water $\left(28^{\circ} \mathrm{C}\right)$ during imaging. Circulation was assessed by transmitted light to confirm vitality of the specimen at the beginning and end of each recording. Analysis was performed in Zen Black software (Zeiss).

\section{Analysis of FDSS data}

An algorithm to track changes in calcium activity using a "moving $\Delta \mathrm{F} / \mathrm{F}_{\mathrm{o}}$ " was devised. The method normalizes the instantaneous average fluorescence for the area of the fish body within the well by the following formula: (average $F_{\text {fish }}(t)-F_{0}$ ) $/ F_{0}$, where $F_{o}=$ average $F_{\text {fish }}$ (averaged over each pixel, for each time sample), and smoothed with a 1000-sample ( 79sec) boxcar moving average. For detecting events, the F/Fo time-series is further smoothed with a 25-sample $(\sim 1.975 \mathrm{sec})$ boxcar moving average. Fish movement is tracked and position, linear velocity, angular velocity estimated. Events are initially detected by identifying peaks that exceeded an empirically determined permissive threshold (>0.05), while the start and end of each event is then identified by the zero-crossing of the smoothed 1st derivative. Subsequently, 47 measurements for each event are obtained related to intensity, distance traveled, linear velocity, angular velocity, total revolutions, and others. Additional measurements for the time-series include total distance, 
maximum/minimum intensity, and total fish size. Calcium events whose max intensity $F / F_{o}$ exceeded 0.1 were retained as significant.

Phosphorylated extracellular signal-related kinase (pERK) staining pERK staining was performed as described elsewhere with minor modifications (Thyme et al., 2019). Briefly, zebrafish larvae were collected and pooled at $5 \mathrm{dpf}$, given $20 \mathrm{~min}$ to acclimate in a quiet room and then were quickly fixed in $4 \%$ Paraformaldehyde (PFA) $+0.25 \%$ Triton $\mathrm{X}$ overnight. Pigmentation was removed by bleaching for 10min in sterile fish water containing $3 \%$ $\mathrm{H}_{2} \mathrm{O} 2,1 \% \mathrm{KOH}$. Samples were washed three times with PBST (PBS with $0.25 \%$ Triton X). Antigen retrieval was performed first by incubation of samples in $150 \mathrm{mM}$ Tris $\mathrm{HCl} \mathrm{pH} 9.0$ for $5 \mathrm{~min}$ followed by $70^{\circ} \mathrm{C}$ for $15 \mathrm{~min}$. Afterwards, they were washed twice with PBST, for $5 \mathrm{~min}$ each. The larvae were then permeabilized by incubation in 0.05\% Trypsin-EDTA on ice for $45 \mathrm{~min}$ and subsequently washed three times in PBST. Samples were incubated in blocking solution containing 2\% Normal Goat Serum, 1\% Bovine Serum Albumin, and 1\% DMSO in PBST for 1h. Larvae were incubated with pERK and tERK antibodies in 1\% BSA and 1\% DMSO in PBST for $48 \mathrm{~h}$ at $4^{\circ} \mathrm{C}$ on a rocker. Primary antibodies: mouse anti-total ERK (p44/p42 MAPK (Erk 1/2) (L34F12) mouse mAb Cell Signaling \#4696) 1:100, rabbit anti-phospho-ERK (Phospho-p44/42 MAPK (Erk1/2) (Thr202/Tyr204) rabbit mAb Cell Signaling \#4370) 1:400. After incubation samples were washed with PBST three times for $15 \mathrm{~min}$ followed by incubation with the secondary antibody solution (donkey anti-rabbit Alexa Fluor 488 (Invitrogen A21206) 1:200, goat antimouse Alexa Fluor 647 (Invitrogen A32728) 1:200) overnight on a rocker at $4^{\circ} \mathrm{C}$. Samples were washed three times, $15 \mathrm{~min}$ each with PBST and stored in PBST at $4^{\circ} \mathrm{C}$ until imaging. Genotypes were determined after imaging. Analysis of pERK-tERK staining ratio was performed as previously described (Thyme et al., 2019).

\section{Confocal Imaging}

Transgenic Tg(dlx6a-1.4kbdlx5a/dlx6a:GFP::vglut2:DsRed) animals (kindly donated by Ellen Hoffman, Yale School of Medicine, New Haven (Hoffman et al., 2016)) were crossed into the transparent mitfa background and then with our pcdh19 mutants, resulting in loss of pigmentation and expression of GFP in inhibitory dlx6/5-positve neurons and dsRed in excitatory vGlut2-postive neurons. Adults of this line were allowed to breed for $1 \mathrm{~h}$ and then all eggs were collected and held at low densities to avoid crowding related maturation delays. Despite the low density, we observed a gradient of maturity in very young larvae, therefore 2dpf larvae used for imaging and analysis were benchmarked based on general developmental milestones (Kimmel et al., 1995) to ensure the use of developmentally matched larvae for experiments. Only hatched larvae that reached at least the long-pec phase with obvious markers of whole-body maturity were included for subsequent brain analysis. Larvae were 4\%PFA fixed at 2dpf or $5 \mathrm{dpf}$ over night for confocal imaging. Fixed larvae were mounted dorsally in $1.2 \%$ agarose and GFP and dsRed fluorescence was imaged immediately after on a Zeiss LSM700 with $15 \mu \mathrm{m}$ step size and a 10x objective lens. For imaging of pcdh19 mosaic mutants, wildtype transgenic zebrafish were bred and pcdh19 sgRNAs or scrambled sgRNA was injected into the 1-cell stage.

\section{TUNEL assay}

Zebrafish larvae were fixed overnight in $4 \% \mathrm{PFA}+0.25 \%$ Triton $\mathrm{X}$ and washed in PBST. Pigmentation was removed with $10 \mathrm{~min}$ bleaching in $3 \% \mathrm{H} 2 \mathrm{O} 2,1 \% \mathrm{KOH}$ in sterile fish water. Samples were incubated in $150 \mathrm{mM}$ Tris $\mathrm{HCl} \mathrm{pH} 9.0$ for $5 \mathrm{~min}$ at room temperature, then at $70^{\circ} \mathrm{C}$ 
for $15 \mathrm{~min}$. Permeabilization was performed in $0.05 \%$ Trypsin-EDTA on ice for $45 \mathrm{~min}$. Afterwards samples were incubated for $2 \mathrm{~h}$ in the In Situ Cell Death Detection Kit (Roche) enzyme labeling solution at $37^{\circ} \mathrm{C}$. Prior to imaging, samples were counterstained in $300 \mathrm{nM}$ DAPI for $30 \mathrm{~min}$.

\section{Image Analysis}

For cell counting in transgenic pcdh19 mutants, z-stacks of confocal images were analyzed with genotypes blinded to the user, using ImageJ software. After background subtraction and noise smoothing, a threshold was determined that includes most cells of the tectum and minimizes background fluorescence. Very bright cells were automatically separated in ImageJ using the watershed function. The average number of all cells counted in the tectum of each zebrafish line was reported. Since background noise was substantial in the dsRed-expressing cell populations, we excluded these from the cell counting analysis.

\section{Western Blot}

For each sample 10-20 zebrafish heads from the same previously genotyped genetic background were pooled in RIPA buffer for assessment of Pcdh19 protein levels. Using a tissue homogenizer, larval heads were homogenized for 35-45s on ice, until the samples was uniformly cloudy. Protein was pelleted by centrifugation at 14000 rpm for $15 \mathrm{~min}$ at $4 \mathrm{C}$ and resuspended in $20 \mu \mathrm{l}$ buffer for subsequent BCA assay to estimate protein concentration. An equal protein amount was used and mixed with $5 \mu \mathrm{l}$ of $4 \mathrm{x}$ Blot sample buffer and DI water and heated for $5 \mathrm{~min}$ at $95^{\circ} \mathrm{C}$. Samples and protein marker were loaded on a SDS-polyacrylamide gel and then blotted on to a PVDF membrane that was pretreated with $100 \%$ methanol for $5 \mathrm{~min}$ and then transferred to $1 \mathrm{x}$ transfer buffer. After successful blotting, membranes were blocked in blocking buffer for 1 h on a shaker. Primary antibodies were applied in blocking buffer at $4^{\circ} \mathrm{C}$ over-night (alpha-tubulin, Abcam, 1:1000 dilution and Pcdh19, Abcam, in 1:200 dilution). After 3 wash steps in PBS-T (o.1\% triton), secondary antibodies IR-Odyssey were applied in blocking buffer in a 1:10000 dilution for $1 \mathrm{~h}$ at room temperature. Washed membranes were imaged with the LiCor Odyssey system.

\section{$\underline{\text { Statistical Analysis }}$}

For all experiments pcdh19 KO lines were compared to WT and pcdh19 mosaics were compared to scrambled injected (and for LFP recordings in addition to scrambled injected mosaics were also compared to WT and injection controls). All statistical analyses were done using Graphpad Prism 8. Values represent the mean \pm SEM. All statistical tests used can be found in the corresponding figure legends. A p-value of < 0.05 was determined as statistically significant. $\mathrm{N}$ numbers can be found in the corresponding figure legends.

\section{Acknowledgments}

Dr. Barbara Robens is supported by the Deutsche Forschungsgemeinschaft (DFG) Germany research fellowship (\#424602704). Dr. McGraw is supported by the CURE Taking Flight Award and NIH/NINDS Ko8NS118107. Dr. Carsten Robens is supported by the Deutsche Forschungsgemeinschaft (DFG) Germany research fellowship (\#421987027). Drs. Rotenberg and Poduri were supported by NIH/NINDS Ro1NS100766 and the Boston Children's Hospital Translational Research Program. Dr. Thyme is supported by NIH/NIMH RooMH110603 and a Klingenstein-Simons Foundation Fellowship Award. We thank Dr. Hyun Yong Koh for his support with the transcriptomic data analysis. We are grateful to Seniha Ipekci, Gessica Truglio, 
Brandon Jones, and other past laboratory members who were involved in the early stages of this project and to the Boston Children's Hospital Aquatic Facility leadership and staff.

\section{Competing interests statement}

Authors declare no competing financial or non-financial interests in relation to the work.

\section{References}

Afrikanova, T., Serruys, A.S., Buenafe, O.E., Clinckers, R., Smolders, I., de Witte, P.A., Crawford, A.D., and Esguerra, C.V. (2013). Validation of the zebrafish pentylenetetrazol seizure model: locomotor versus electrographic responses to antiepileptic drugs. PLoS One 8, e54166. Ashburner, M., Ball, C.A., Blake, J.A., Botstein, D., Butler, H., Cherry, J.M., Davis, A.P., Dolinski, K., Dwight, S.S., Eppig, J.T., et al. (2000). Gene ontology: tool for the unification of biology. The Gene Ontology Consortium. Nat Genet 25, 25-29.

Baraban, S.C., Dinday, M.T., and Hortopan, G.A. (2013). Drug screening in Scn1a zebrafish mutant identifies clemizole as a potential Dravet syndrome treatment. Nat Commun 4, 2410. Bassani, S., Cwetsch, A.W., Gerosa, L., Serratto, G.M., Folci, A., Hall, I.F., Mazzanti, M., Cancedda, L., and Passafaro, M. (2018). The female epilepsy protein PCDH19 is a new GABAAR-binding partner that regulates GABAergic transmission as well as migration and morphological maturation of hippocampal neurons. Hum Mol Genet 27, 1027-1038.

Blevins, C.J., Emond, M.R., Biswas, S., and Jontes, J.D. (2011). Differential expression, alternative splicing, and adhesive properties of the zebrafish delta1-protocadherins. Neuroscience 199, 523-534.

Cooper, S.R., Emond, M.R., Duy, P.Q., Liebau, B.G., Wolman, M.A., and Jontes, J.D. (2015). Protocadherins control the modular assembly of neuronal columns in the zebrafish optic tectum. J Cell Biol 211, 807-814.

Darland, T., and Dowling, J.E. (2001). Behavioral screening for cocaine sensitivity in mutagenized zebrafish. Proc Natl Acad Sci U S A 98, 11691-11696.

Dentici, M.L., Alesi, V., Quinodoz, M., Robens, B., Guerin, A., Lebon, S., Poduri, A., Travaglini, L., Graziola, F., Afenjar, A., et al. (2021). Biallelic variants in ZNF526 cause a severe neurodevelopmental disorder with microcephaly, bilateral cataract, epilepsy and simplified gyration. J Med Genet.

Depienne, C., Bouteiller, D., Keren, B., Cheuret, E., Poirier, K., Trouillard, O., Benyahia, B., Quelin, C., Carpentier, W., Julia, S., et al. (2009). Sporadic infantile epileptic encephalopathy caused by mutations in PCDH19 resembles Dravet syndrome but mainly affects females. PLoS Genet 5, e1000381.

Depienne, C., and LeGuern, E. (2012). PCDH19-related infantile epileptic encephalopathy: an unusual X-linked inheritance disorder. Human mutation 33, 627-634.

Dibbens, L.M., Tarpey, P.S., Hynes, K., Bayly, M.A., Scheffer, I.E., Smith, R., Bomar, J., Sutton, E., Vandeleur, L., Shoubridge, C., et al. (2008). X-linked protocadherin 19 mutations cause female-limited epilepsy and cognitive impairment. Nat Genet 4O, 776-781.

Dimova, P.S., Kirov, A., Todorova, A., Todorov, T., and Mitev, V. (2012). A novel PCDH19 mutation inherited from an unaffected mother. Pediatric neurology 46, 397-400. 
Duszyc, K., Terczynska, I., and Hoffman-Zacharska, D. (2015). Epilepsy and mental retardation restricted to females: X-linked epileptic infantile encephalopathy of unusual inheritance. J Appl Genet 56, 49-56.

Eimon, P.M., Ghannad-Rezaie, M., De Rienzo, G., Allalou, A., Wu, Y., Gao, M., Roy, A., Skolnick, J., and Yanik, M.F. (2018). Brain activity patterns in high-throughput electrophysiology screen predict both drug efficacies and side effects. Nat Commun 9, 219.

Epi4K Consortium, and Epilepsy Phenome/Genome Project (2017). Ultra-rare genetic variation in common epilepsies: a case-control sequencing study. Lancet Neurol 16, 135-143.

Griffin, A., Carpenter, C., Liu, J., Paterno, R., Grone, B., Hamling, K., Moog, M., Dinday, M.T., Figueroa, F., Anvar, M., et al. (2021). Phenotypic analysis of catastrophic childhood epilepsy genes. Commun Biol 4, 680.

Griffin, A., Hamling, K.R., Knupp, K., Hong, S., Lee, L.P., and Baraban, S.C. (2017). Clemizole and modulators of serotonin signalling suppress seizures in Dravet syndrome. Brain 140, 669-683. Griffin, C., Liu, Paterno, Grone, Hamling, Moog, Dinday, Figueroa, Anvar, Ononuju, Qu, Baraban (2021). Phenotypic analysis of catastrophic childhood epilepsy genes: The Epilepsy Zebrafish Project. BioRxiv.

Grone, B.P., Marchese, M., Hamling, K.R., Kumar, M.G., Krasniak, C.S., Sicca, F., Santorelli, F.M., Patel, M., and Baraban, S.C. (2016). Epilepsy, Behavioral Abnormalities, and Physiological Comorbidities in Syntaxin-Binding Protein 1 (STXBP1) Mutant Zebrafish. PLoS One 11, e0151148. Hayashi, S., Inoue, Y., Hattori, S., Kaneko, M., Shioi, G., Miyakawa, T., and Takeichi, M. (2017). Loss of X-linked Protocadherin-19 differentially affects the behavior of heterozygous female and hemizygous male mice. Sci Rep 7, 5801.

Hoffman, E.J., Turner, K.J., Fernandez, J.M., Cifuentes, D., Ghosh, M., Ijaz, S., Jain, R.A., Kubo, F., Bill, B.R., Baier, H., et al. (2016). Estrogens Suppress a Behavioral Phenotype in Zebrafish Mutants of the Autism Risk Gene, CNTNAP2. Neuron 89, 725-733.

Hortopan, G.A., Dinday, M.T., and Baraban, S.C. (2010). Spontaneous seizures and altered gene expression in GABA signaling pathways in a mind bomb mutant zebrafish. J Neurosci 3O, 1371813728.

Hoshina, N., Johnson-Venkatesh, E.M., Hoshina, M., and Umemori, H. (2021). Female-specific synaptic dysfunction and cognitive impairment in a mouse model of PCDH19 disorder. Science 372.

Hunt, R.F., Hortopan, G.A., Gillespie, A., and Baraban, S.C. (2012). A novel zebrafish model of hyperthermia-induced seizures reveals a role for TRPV4 channels and NMDA-type glutamate receptors. Exp Neurol 237, 199-206.

Kimmel, C.B., Ballard, W.W., Kimmel, S.R., Ullmann, B., and Schilling, T.F. (1995). Stages of embryonic development of the zebrafish. Dev Dyn 203, 253-310.

Kolc, K.L., Sadleir, L.G., Scheffer, I.E., Ivancevic, A., Roberts, R., Pham, D.H., and Gecz, J. (2018). A systematic review and meta-analysis of 271 PCDH19-variant individuals identifies psychiatric comorbidities, and association of seizure onset and disease severity. Mol Psychiatry.

Kysil, E.V., Meshalkina, D.A., Frick, E.E., Echevarria, D.J., Rosemberg, D.B., Maximino, C., Lima, M.G., Abreu, M.S., Giacomini, A.C., Barcellos, L.J.G., et al. (2017). Comparative Analyses of Zebrafish Anxiety-Like Behavior Using Conflict-Based Novelty Tests. Zebrafish 14, 197-208.

Lin, X., Duan, X., Jacobs, C., Ullmann, J., Chan, C.Y., Chen, S., Cheng, S.H., Zhao, W.N., Poduri, A., Wang, X., et al. (2018). High-throughput brain activity mapping and machine learning as a foundation for systems neuropharmacology. Nat Commun 9, 5142. 
Liu, J., and Baraban, S.C. (2019). Network Properties Revealed during Multi-Scale Calcium Imaging of Seizure Activity in Zebrafish. eNeuro 6.

Livak, K.J., and Schmittgen, T.D. (2001). Analysis of relative gene expression data using real-time quantitative PCR and the 2(-Delta Delta C(T)) Method. Methods 25, $402-408$.

Meeker, N.D., Hutchinson, S.A., Ho, L., and Trede, N.S. (2007). Method for isolation of PCRready genomic DNA from zebrafish tissues. Biotechniques 43, 610, 612, 614.

Mincheva-Tasheva, S., Nieto Guil, A.F., Homan, C.C., Gecz, J., and Thomas, P.Q. (2021). Disrupted Excitatory Synaptic Contacts and Altered Neuronal Network Activity Underpins the Neurological Phenotype in PCDH19-Clustering Epilepsy (PCDH19-CE). Mol Neurobiol 58, 20052018.

Pederick, D.T., Homan, C.C., Jaehne, E.J., Piltz, S.G., Haines, B.P., Baune, B.T., Jolly, L.A., Hughes, J.N., Gecz, J., and Thomas, P.Q. (2016). Pcdh19 Loss-of-Function Increases Neuronal Migration In Vitro but is Dispensable for Brain Development in Mice. Sci Rep 6, 26765.

Pederick, D.T., Richards, K.L., Piltz, S.G., Kumar, R., Mincheva-Tasheva, S., Mandelstam, S.A., Dale, R.C., Scheffer, I.E., Gecz, J., Petrou, S., et al. (2018). Abnormal Cell Sorting Underlies the Unique X-Linked Inheritance of PCDH19 Epilepsy. Neuron 97, 59-66 e55.

Rakotomamonjy, J., Sabetfakhri, N.P., McDermott, S.L., and Guemez-Gamboa, A. (2020). Characterization of seizure susceptibility in Pcdh19 mice. Epilepsia 61, 2313-2320.

Samanta, D. (2020). PCDH19-Related Epilepsy Syndrome: A Comprehensive Clinical Review. Pediatr Neurol 105, 3-9.

Schnorr, S.J., Steenbergen, P.J., Richardson, M.K., and Champagne, D.L. (2012). Measuring thigmotaxis in larval zebrafish. Behav Brain Res 228, 367-374.

Smith, L., Singhal, N., El Achkar, C.M., Truglio, G., Rosen Sheidley, B., Sullivan, J., and Poduri, A. (2018). PCDH19-related epilepsy is associated with a broad neurodevelopmental spectrum. Epilepsia.

Thyme, S.B., Pieper, L.M., Li, E.H., Pandey, S., Wang, Y., Morris, N.S., Sha, C., Choi, J.W., Herrera, K.J., Soucy, E.R., et al. (2019). Phenotypic Landscape of Schizophrenia-Associated Genes Defines Candidates and Their Shared Functions. Cell 177, 478-491 e420.

Turrini, L., Fornetto, C., Marchetto, G., Mullenbroich, M.C., Tiso, N., Vettori, A., Resta, F., Masi, A., Mannaioni, G., Pavone, F.S., et al. (2017). Optical mapping of neuronal activity during seizures in zebrafish. Sci Rep 7, 3025 .

Wang, W., and Frankel, W.N. (2021). Overlaps, gaps, and complexities of mouse models of Developmental and Epileptic Encephalopathy. Neurobiol Dis 148, 105220.

$\mathrm{Wu}, \mathrm{Q}$. (2005). Comparative genomics and diversifying selection of the clustered vertebrate protocadherin genes. Genetics 169, 2179-2188. 